\title{
A Direct Numerical Simulation analysis of turbulent V-shaped flames propagating into droplet-laden mixtures
}

Gulcan Ozel Erol ${ }^{1, *}$, Josef Hasslberger², Markus Klein², Nilanjan Chakraborty ${ }^{1}$

${ }^{1}$ School of Engineering, Newcastle University, Newcastle-Upon-Tyne, NE17RU, United Kingdom.

${ }^{2}$ Bundeswehr University Munich, Werner-Heisenberg-Weg 39, 85577 Neubiberg, Germany.

* Corresponding author: G.Ozel-Erol2@newcastle.ac.uk 


\section{ABSTRACT}

Three-dimensional Direct Numerical Simulations (DNS) data has been used to analyse the gaseous phase combustion behaviour in a V-shaped flame configuration where fuel is supplied in the form of droplets such that an overall (i.e. liquid+gaseous) equivalence ratio of unity is maintained in the unburned gas. The analysis has been carried out for different initially mono-sized droplet diameters. A gaseous premixed V-shaped flame with the same flow conditions has been utilised to compare the flame propagation in $\mathrm{V}$-shaped spray flames with a corresponding gaseous premixed flame case. It has been found that combustion in the gaseous phase mainly takes place under fuel-lean mode and the probability of finding fuel-lean burning increases with increasing droplet diameter. However, the mean equivalence ratio of the predominantly fuel-lean mixture increases with increasing axial distance from the flame holder. The presence of droplets has been found to give rise to dimples on the reaction progress variable isosurfaces, and the effects of droplet-induced flame wrinkling are reflected by the widening of the curvature probability density functions for large droplets. The heat release has been found to arise mainly from the premixed mode of combustion. Detailed analysis of reaction progress variable transport has been used to explain the mean variations of consumption speed and density-weighted displacement speed with the axial distance from the flame holder. The mean values of consumption speed, densityweighted displacement speed and turbulent burning velocity have been found to decrease with increasing droplet diameter. The flame speed statistics have been utilised to explain that the enhancement of the burning rate cannot be equated to the enhancement of the flame surface area in turbulent droplet-laden V-shaped flames.

Keywords: Droplet combustion, V-shaped flame, Equivalence ratio, Displacement speed, Consumption speed, Direct Numerical Simulations 


\section{INTRODUCTION}

The V-shaped flame is one of the most commonly used laboratory-scale configurations, which has been extensively investigated in previous experimental (Smith and Gouldin, 1978; Veynante et al., 1994, 1996; Gouldin, 1996; Shepherd, 1996; Sattler et al., 2002; Vena et al., 2011, 2015; Sponfeldner et al., 2015; Letty et al., 2013) and numerical (Alshaalan and Rutland, 1998; Domingo et al., 2005; Bell et al., 2005; Wu et al., 2011; Dunstan et al., 2011, 2012) studies. Smith and Gouldin (1978) proposed an experimental methodology to measure turbulent flame speed in V-shaped turbulent methane-air flames with different equivalence ratios and turbulence intensities. Veynante et al. $(1994,1996)$ used experimental data of turbulent premixed propane-air V-flames to analyse flame surface density transport statistics. Gouldin (1996) and Shepherd (1996) investigated the burning and flame propagation rates by measuring the flame surface area in turbulent premixed V-shaped flames. Shepherd (1996) compared a stagnation point flame with a V-shaped flame and identified important differences in flame structure and burning rate between the two flames. A similar conclusion was drawn by Dunstan et al. (2011) based on Direct Numerical Simulations (DNS). Sattler et al. (2002) observed a linear increase in the mean flamelet area with downstream distance by taking measurements at several axial distances from the stabilizing rod for turbulent premixed methane/air V-flames. The flame topology and flame brush thickness for turbulent V-flames at different turbulence intensities have been experimentally investigated by Kheirkhah and Gulder (2014). Vena et al. (2011,2015) experimentally analysed flame topology and heat release rate statistics for turbulent V-flames for stratified iso-octane/air mixtures. It was shown by Vena et al. (2011) that equivalence ratio gradients have significant effects on flame wrinkling in comparison to the corresponding fully premixed reference flame. Sponfeldner et al. (2015) concentrated on the statistical behaviours of principal strain-rates and the local flamelet normal vector in their experimental analysis of premixed turbulent V-flames. 
Advancements of high-performance computing enabled detailed and parametric analyses of $\mathrm{V}$ shaped flames using DNS. Alshaalan and Rutland (1998) performed a three-dimensional DNS analysis with a single-step chemical mechanism to analyse the adiabatic and non-adiabatic flame-wall interactions in a V-flame configuration. Domingo et al. (2005) utilised twodimensional DNS and Large Eddy Simulations (LES) of turbulent premixed V-flames for development and validation of Flame Surface Density-Probability Density Function (FSDPDF) closures in the context of LES. Bell et al. (2005) carried out three-dimensional DNS for a laboratory-scale turbulent premixed V-shaped flame and reported a good agreement in the mean flame structure and the mean axial and transverse velocities between DNS results and experimental data. Wu et al. (2011) investigated the ignition and propagation of a V-shaped premixed ethylene-air turbulent flame using LES and an increase in flame angle is observed relative to the laminar cases. Dunston et al. $(2011,2012)$ performed an extensive threedimensional DNS analysis with single-step chemistry for premixed turbulent V-flames under different turbulence intensities. The expression derived for the turbulent displacement speed showed a similar trend with the turbulent consumption speed in the downstream of the flame. However, qualitative differences between the turbulent displacement and consumption speeds originate due to turbulent and molecular diffusion (Dunstan et al., 2011).

Despite significant practical relevance of turbulent combustion of droplet-laden mixtures, the V-flame configuration has rarely been used (Letty et al., 2013) for the analysis of spray combustion. Although several previous analytical (Polymeropoulos, 1984; Silverman et al., 1993; Greenberg et al., 1993), experimental (Burgoyne and Cohen, 1954; Mizutani and Nishimoto, 1972; Hayashi et al., 1977; Szekely and Faeth, 1983; Ballal and Lefebvre, 1981; Lawes and Saat, 2011) and computational (Reveillon and Vervisch, 2000; Nakamura et al., 
2005; Wang and Rutland, 2005; Watanabe et al., 2007; Reveillon and Demoulin, 2007; Sreedhara and Huh, 2007; Xia and Luo, 2010; Fujita et al., 2013; Schroll et al., 2009; Wandel et al., 2009; Wandel, 2014; Neophytou et al., 2010, 2012; Wacks et al., 2016; Wacks and Chakraborty, 2016; Ozel Erol et al., 2018, 2019a, 2019b) studies investigated flame propagation in droplet-laden mixtures in conventional canonical configurations (e.g. statistically planar and spherical flames), a relatively limited effort (Letty et al., 2013) has been directed to the investigations on spray flames in relatively more complex laboratory-scale configurations such as V-shaped flames. Letty et al. (2013) carried out experiments to understand the combustion behaviour of V-shaped flames in heptane droplet-laden mixtures. They compared temperature distributions between droplets and gaseous phase mixture and indicated that the presence of droplets significantly alters the temperature of the gaseous mixture. However, the flame propagation statistics for droplet-laden mixtures in a V-shaped flame configuration are still incompletely understood due to the complex interaction between droplets, flame and the background fluid flow. To address this gap in the existing literature, three-dimensional carrier phase Direct Numerical Simulations (DNS) with modified single-step chemistry (Ozel-Erol et al., 2019b) have been performed in this analysis for three different droplet diameters for an overall (liquid+gaseous) equivalence ratio of unity in the unburned droplet-laden mixture. The variations of gaseous equivalence ratio $\phi_{g}$, flame curvature $\kappa_{m}$ and flame displacement and consumption speeds have been presented at different axial locations, which have been explained based on physical principles. In this respect, the main objectives of this paper are:

1. To demonstrate the evolution of the gaseous reacting mixture composition within the flame at different axial locations from the flame holder for $\mathrm{V}$-shaped flames propagating in dropletladen mixtures.

2. To explain the influences of droplet diameter on the reaction zone structure and flame propagation characteristics at different axial locations from the flame holder and to explain the 
differences in flame structure arising from supplying the fuel in liquid form by comparing to a turbulent gaseous stoichiometric premixed V-shaped flame.

3. To indicate implications of the above findings in the context of modelling of turbulent combustion of droplet-laden mixtures.

The mathematical background and numerical implementation pertaining to this analysis are presented in the next section. This will be followed by the presentation of the results and their subsequent discussion. The main findings are summarised and conclusions are drawn in the final section of this paper.

\section{MATHEMATICAL BACKGROUND}

A modified single-step Arrhenius-type chemical mechanism (Tarrazo et al., 2006) has been adopted to keep the computational demand within reasonable limits. This chemical mechanism provides a realistic $\phi_{g}$ dependence of the unstrained laminar burning velocity $S_{b\left(\phi_{g}\right)}$ and adiabatic flame temperature $T_{a d\left(\phi_{g}\right)}$ in hydrocarbon-air flames. For further information on this behaviour in hydrocarbon-air flames, the interested readers are directed to Malkeson and Chakraborty (2010). Comparisons between the laminar flame speed of n-heptane from experiments (Kumar et al., 2007), simulations with detailed chemical mechanism (Chaos et al., 2007) and modified single step chemistry used in this study are presented in Fig 1 . The variations of $S_{b\left(\phi_{g}\right)} /\left\{S_{b\left(\phi_{g}\right)}\right\}_{\max }$ and $T_{a d\left(\phi_{g}\right)}$ with $\phi_{g}$ for the present thermo-chemistry have been found to exhibit good agreements with previous experimental data (Swaminathan and Bray, 2011) and the results obtained from a detailed chemical mechanism. Note that, single step chemistry has been used to analyse the turbulent spray flames in several previous studies (Reveillon and Demoulin, 2007; Sreedhara and Huh, 2007; Xia and Luo; 2010; Wang and 
Rutland, 2005; Wandel, 2014) which offered valuable physical insights while being consistent with experimental observations. For example, the findings of simple chemistry DNS by OzelErol et al. $(2018,2019)$ have been found to be qualitatively consistent with the experimental findings (Hayashi et al., 1977; Lawes and Saat,2011). To date, the majority of the DNS based analyses on droplet combustion have been conducted using simple chemical mechanisms (Nakamura et al., 2005; Reveillon and Vervisch, 2005; Wang and Rutland, 2005; Reveillon and Demoulin, 2007; Schroll et al., 2009; Watanabe et al., 2007; Sreedhara and Huh, 2007; Wandel et al., 2009; Wandel, 2014; Wacks et al., 2016; Haruki et al., 2018; Pillai and Kurose, 2018, 2019). It is also worth noting that it has been demonstrated in several previous analyses that the flame propagation statistics extracted from simple chemistry DNS (Chakraborty and Cant, 2004, 2005; Jenkins et al., 2006; Klein et al., 2016; Chakraborty, 2007; Han and Huh.,2008) are at least qualitatively similar to those obtained from detailed chemistry DNS (Echekki and Chen, 1996; Chen and Im, 1998; Peters et al., 1998; Hawkes and Chen, 2004; Hawkes and Chen, 2005; Chakraborty et al., 2008) data.

(a)

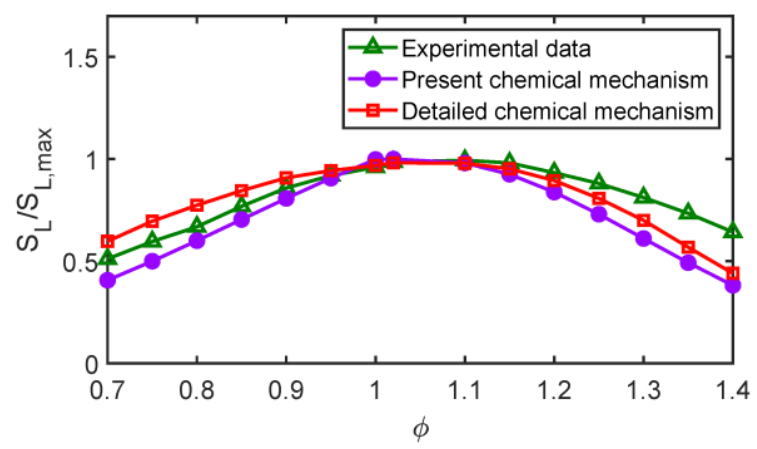

(b)

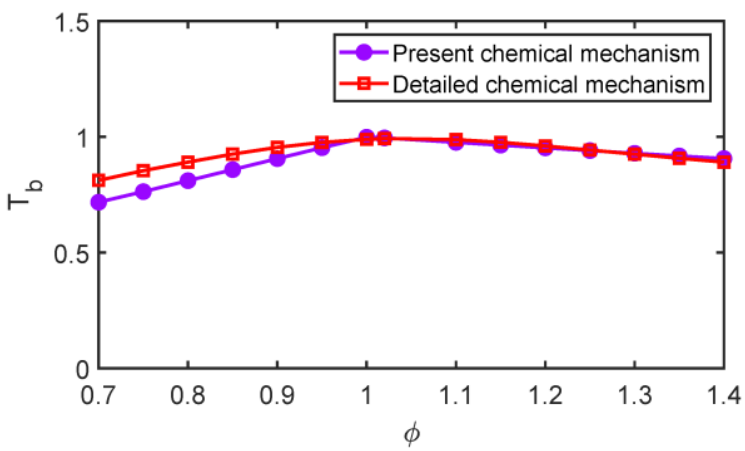

Figure 1. Variations of the (a) normalised laminar burning velocity $S_{b\left(\phi_{g}\right)} /\left\{S_{b\left(\phi_{g}\right)}\right\}_{\max }$ and (b) normalised adiabatic flame temperature $T_{b\left(\phi_{g}\right)}=\left(T_{a d\left(\phi_{g}\right)}-T_{0}\right) /\left(T_{a d\left(\phi_{g=1}\right)}-T_{0}\right)$ with equivalence ratio $\phi_{g}$ for $n$-heptane obtained from modified single step chemistry (Tarrazo et al., 2006), detailed chemical mechanism (Chaos et al., 2007) and experimental (Kumar et al., 2007) data. 
In this analysis, the Lewis numbers of all species are assumed to be unity and all species in the gaseous phase are taken to be perfect gases. Standard values have been considered for the ratio of specific heats $\left(\gamma=C_{p}^{g} / C_{v}^{g}=1.4\right.$, where $C_{p}^{g}$ and $C_{v}^{g}$ are the gaseous specific heats at constant pressure and constant volume respectively) and Prandtl number $\left(\operatorname{Pr}=\mu C_{p}^{g} / \lambda=0.7\right.$ where $\mu$ is the dynamic viscosity and $\lambda$ is the thermal conductivity of the gaseous phase). The position $\vec{x}_{d}$, velocity $\vec{u}_{d}$, diameter $a_{d}$ and temperature $T_{d}$ of the individual droplets are transported in the following manner using a Lagrangian approach (Reveillon and Vervisch, 2000; Reveillon and Demoulin, 2007; Schroll et al., 2009; Wandel et al., 2009; Wandel, 2014; Neophytou et al., 2010, 2012; Wacks et al., 2016; Wacks and Chakraborty, 2016; Ozel Erol et al., 2018, 2019a, 2019b):

$$
\frac{d \vec{x}_{d}}{d t}=\vec{u}_{d} ; \frac{d \vec{u}_{d}}{d t}=\frac{\vec{u}\left(\vec{x}_{d}, t\right)-\vec{u}_{d}}{\tau_{d}^{u}} ; \frac{d a_{d}^{2}}{d t}=-\frac{a_{d}^{2}}{\tau_{d}^{p}} ; \frac{d T_{d}}{d t}=\frac{\widehat{T}\left(\vec{x}_{d}, t\right)-T_{d}-B_{d} L_{v} / C_{P}^{g}}{\tau_{d}^{T}}
$$

In the transport equations, $\widehat{T}$ is the gaseous phase temperature, $L_{v}$ is the latent heat of vaporization and $\tau_{d}^{u}, \tau_{d}^{p}$ and $\tau_{d}^{T}$ stand for the relaxation timescales for droplet velocity, diameter and temperature, respectively, which can be expressed as (Reveillon and Vervisch, 2000; Reveillon and Demoulin, 2007):

$$
\tau_{d}^{u}=\frac{\rho_{d} a_{d}^{2}}{\left(18 C_{u} \mu\right)} ; \tau_{d}^{p}=\frac{\rho_{d} a_{d}^{2}}{4 \mu} \frac{S c}{S h_{c} \ln \left(1+B_{d}\right)} ; \tau_{d}^{T}=\frac{\rho_{d} a_{d}^{2}}{6 \mu} \frac{P r}{N u_{c}} \frac{B_{d}}{\ln \left(1+B_{d}\right)} \frac{C_{p}^{L}}{C_{p}^{g}}
$$

where, $\rho_{d}$ is the droplet density, $S c$ is the Schmidt number, $C_{p}^{L}$ is the specific heat for the liquid phase. The liquid fuel properties used in the analysis are summarised in Table 1. In Eq. $2, C_{u}=$ $1+R e_{d}^{2 / 3} / 6$ is the corrected drag coefficient, $R e_{d}$ is the droplet Reynolds number, $B_{d}$ is the Spalding number, $S h_{c}$ is the corrected Sherwood number and $N u_{c}$ is the corrected Nusselt number, which are defined as (Reveillon and Vervisch, 2000; Reveillon and Demoulin, 2007):

$$
R e_{d}=\frac{\rho\left|\vec{u}\left(\vec{x}_{d}, t\right)-\vec{u}_{d}\right| a_{d}}{\mu} ; B_{d}=\frac{\left[Y_{F}^{S}-Y_{F}\left(\vec{x}_{d}, t\right)\right]}{\left(1-Y_{F}^{S}\right)} ;
$$




$$
S h_{c}=N u_{c}=2+\frac{0.555 R e_{d} S c}{\left(1.232+R e_{d} S c^{4 / 3}\right)^{1 / 2}}
$$

where $Y_{F}^{S}$ is the value of fuel mass fraction $Y_{F}$ at the droplet surface. The Clausius-Clapeyron equation is used to evaluate the partial pressure of the fuel vapour at the droplet surface $p_{F}^{S}$, which is in turn used to calculate the Spalding number $B_{d}$, which leads to the following expressions (Reveillon and Vervisch, 2000; Reveillon and Demoulin, 2007):

$$
p_{F}^{S}=p_{\text {ref }} \exp \left(\frac{L_{v}}{R}\left[\frac{1}{T_{\text {ref }}^{S}}-\frac{1}{T_{d}^{S}}\right]\right) ; Y_{F}^{S}=\left(1+\frac{W_{g}}{W_{F}}\left[\frac{p\left(\vec{x}_{d}, t\right)}{p_{F}^{S}}-1\right]\right)^{-1}
$$

where $R$ is the gas constant, $T_{r e f}^{s}$ is the boiling point of the fuel at a pressure $p_{\text {ref }}$ and $T_{d}^{S}$ is assumed to be $T_{d}$, and $W_{g}$ and $W_{F}$ are the molecular weights of gaseous mixture and fuel, respectively. The liquid fuel droplets are coupled with the surrounding gaseous mixture with additional source terms in the gaseous transport equations as follows (Schroll et al., 2009; Wandel et al., 2009; Wandel, 2014; Neophytou et al., 2010, 2012; Wacks et al., 2016; Wacks and Chakraborty, 2016; Ozel Erol et al., 2018, 2019a, 2019b):

$$
\frac{\partial(\rho \varphi)}{\partial t}+\frac{\partial\left(\rho u_{j} \varphi\right)}{\partial x_{j}}=\frac{\partial}{\partial x_{j}}\left[R_{\varphi} \frac{\partial \varphi_{1}}{\partial x_{j}}\right]+\dot{\omega}_{\varphi}+\dot{S}_{g}+\dot{S}_{\varphi}
$$

where $\varphi=\left\{1, u_{i}, e, Y_{F}, Y_{O}\right\}$ and $\varphi_{1}=\left\{1, u_{i}, \widehat{T}, Y_{F}, Y_{O}\right\}$ for the conservation equations of mass, momentum, energy, and mass fractions, respectively and $R_{\varphi}=\rho v / \sigma_{\varphi}$ is the generalised diffusion coefficient associated with $\varphi=\left\{1, u_{i}, Y_{F}, Y_{O}\right\}$ whereas $R_{\varphi}$ is given by $R_{\varphi}=\lambda$ for $\varphi=$ $e$, and $u_{i}$ is the fluid velocity in the $i^{\text {th }}$ direction. The term $\dot{\omega}_{\varphi}$ corresponds to the reaction rate contribution, $\dot{S}_{g}$ refers to an appropriate gaseous phase source term and $\dot{S}_{\varphi}$ is the appropriate source term due to the coupling between Lagrangian particulate and Eulerian carrier phases, which is tri-linearly interpolated from the droplet's sub-grid position, $\vec{x}_{d}$, to the eight surrounding nodes. Here, $v$ is the kinematic viscosity, and $\sigma_{\varphi}$ is an appropriate Schmidt number corresponding to $\varphi$. 
A reaction progress variable $c$, based on oxygen mass fraction, $Y_{O}$ and mixture fraction, $\xi=$ $\left(Y_{F}-Y_{O} / s+Y_{O \infty} / s\right) /\left(Y_{F \infty}+Y_{O \infty} / s\right)$, can be written in the following manner (Wacks et al., 2016; Wacks and Chakraborty, 2016):

$$
c=\frac{\left[(1-\xi) Y_{O \infty}-Y_{O}\right]}{\left[(1-\xi) Y_{O \infty}-\max \left(0,\left\{\xi_{s t}-\xi\right\} / \xi_{s t}\right) Y_{O \infty}\right]}
$$

where $Y_{O \infty}=0.233$ is the oxygen mass fraction in air and $Y_{F \infty}=1.0$ is the fuel mass fraction in the pure fuel stream. Considering n-heptane, $\mathrm{C}_{7} \mathrm{H}_{16}$ as the fuel in the analysis, $s=3.52$ denotes the stoichiometric mass ratio of oxygen to fuel and $Y_{F s t}=0.0621$ and $\xi_{s t}=0.0621$ represent the corresponding stoichiometric fuel mass fraction and stoichiometric mixture fraction, respectively.

The transport equation of the reaction progress variable, $c$ is given by (Wacks et al., 2016; Wacks and Chakraborty, 2016; Ozel Erol et al., 2018, 2019a, 2019b):

$$
\rho \frac{\partial c}{\partial t}+\rho u_{j} \frac{\partial c}{\partial x_{j}}=\nabla \cdot(\rho D \nabla c)+\dot{w}_{c}+\dot{S}_{l i q, c}+\dot{A}_{c}
$$

where $D$ is the molecular diffusivity, $\dot{w}_{c}$ indicates the reaction rate of reaction progress variable, $\dot{S}_{\text {liq, },}$ represents the source/sink term arising due to droplet evaporation and $\dot{A}_{c}$ is the crossscalar dissipation term arising due to mixture inhomogeneity. The reaction rate of the reaction progress variable $\dot{w}_{c}$ can be expressed as (Wacks et al., 2016; Wacks and Chakraborty, 2016; Ozel Erol et al., 2018, 2019a, 2019b):

$$
\dot{w}_{c}=-\xi_{s t} \dot{w}_{O} /\left[Y_{O \infty} \xi\left(1-\xi_{s t}\right)\right] \text { for } \xi \leq \xi_{s t} \text { and } \dot{w}_{c}=-\dot{w}_{O} /\left[Y_{O \infty}(1-\xi)\right] \text { for } \xi>\xi_{s t}
$$

where $\dot{w}_{O}$ is the reaction rate of oxygen (Wacks et al., 2016; Wacks and Chakraborty, 2016). The term associated with droplet evaporation $\dot{S}_{l i q, c}$ is calculated as (Wacks et al., 2016; Wacks and Chakraborty, 2016; Ozel Erol et al., 2018, 2019a, 2019b): 


$$
\begin{aligned}
& \dot{S}_{l i q, c}=-\xi_{s t}\left[\xi \dot{S}_{O}+\left(Y_{O \infty}-Y_{O}\right) \dot{S}_{\xi}\right] /\left[Y_{O \infty} \xi^{2}\left(1-\xi_{s t}\right)\right] \text { for } \xi \leq \xi_{s t} \\
& \text { and } \dot{S}_{l i q}=-\left[(1-\xi) \dot{S}_{O}+Y_{O} \dot{S}_{\xi}\right] /\left[Y_{O \infty}(1-\xi)^{2}\right] \text { for } \xi>\xi_{s t}
\end{aligned}
$$

where $\dot{S}_{\xi}=\left(\dot{S}_{F}-\dot{S}_{O} / s\right) /\left(Y_{F \infty}+Y_{O \infty} / s\right)$ is the droplet source/sink term in the mixture fraction transport equation, $\dot{S}_{F}=\Gamma_{m}\left(1-Y_{F}\right)$ and $\dot{S}_{O}=-\Gamma_{m} Y_{O}$ are the droplet source/sink terms in the mass fraction transport equations for fuel and oxygen, respectively and $\Gamma_{m}$ is the source term in the mass conservation equation due to evaporation (Wacks et al., 2016; Wacks and Chakraborty, 2016). The term associated with mixture inhomogeneity $\dot{A}_{c}$ is given as (Wacks et al., 2016; Wacks and Chakraborty, 2016):

$$
\dot{A}_{c}=2 \rho D \nabla \xi \cdot \nabla c / \xi \text { for } \xi \leq \xi_{s t} \text { and } \dot{A}_{c}=-2 \rho D \nabla \xi \cdot \nabla c /(1-\xi) \text { for } \xi>\xi_{s t}
$$

Alternatively, Eq. 7 can be rearranged in the kinematic form for a given $c$ isosurface as (Wacks et al., 2016; Wacks and Chakraborty, 2016):

$$
\frac{\partial c}{\partial t}+u_{j} \frac{\partial c}{\partial x_{j}}=S_{d}|\nabla c|
$$

where $S_{d}$ represents the displacement speed, which can be expressed as (Wacks et al., 2016; Wacks and Chakraborty, 2016):

$$
S_{d}=\frac{\left[\nabla \cdot(\rho D \nabla c)+\dot{w}_{c}+\dot{S}_{l i q, c}+\dot{A}_{c}\right]}{\rho|\nabla c|}
$$

Using Eq. 12, the density-weighted displacement speed can be defined as $S_{d}^{*}=\rho S_{d} / \rho_{0}$ where $\rho_{0}$ is the unburned gas density. Another alternative flame speed, known as the consumption speed $S_{c}$, can be defined as (Ozel Erol et al., 2019b; Poinsot et al., 1992; Haworth and Poinsot, 1992):

$$
S_{c}=\rho_{0}^{-1} \int \dot{w}_{c} d n
$$

where $d n$ is the elemental distance in the local flame normal direction. 
Table 1. Liquid fuel properties used in this analysis

\begin{tabular}{|l|l|}
\hline Liquid specific heat at constant pressure, $C_{p}^{l}$ & $2296.45 \mathrm{~J} / \mathrm{kgK}$ \\
\hline Latent heat of vaporization, $L_{v}$ & $315 \mathrm{~kJ} / \mathrm{kg}$ \\
\hline Liquid density, $\rho_{d}$ & $684 \mathrm{~kg} / \mathrm{m}^{3}$ \\
\hline Liquid temperature at the inlet, $T_{0}$ & $300 \mathrm{~K}$ \\
\hline
\end{tabular}

\section{NUMERICAL IMPLEMENTATION}

Simulations of the $\mathrm{V}$-shaped flames propagating in droplet-laden mixtures are carried out using a three-dimensional compressible code SENGA+ (Schroll et al., 2009; Wandel et al., 2009; Wandel, 2014; Neophytou et al., 2010, 2012; Wacks et al., 2016; Wacks and Chakraborty, 2016; Ozel Erol et al., 2018, 2019a, 2019b). The governing equations of mass, momentum, energy and species of the gaseous phase are spatially discretised using a $10^{\text {th }}$ order central difference scheme for the internal grid points, but the order of differentiation gradually decreases to a one-sided $2^{\text {nd }}$ order scheme at the non-periodic boundaries. The time advancement is achieved by employing an explicit low-storage $3^{\text {rd }}$ order Runge-Kutta scheme (Wray, 1990). Boundary conditions in the stream-wise direction ( $x$-direction) are considered to be inflow and outflow according to the Navier-Stokes Characteristic Boundary Conditions (NSCBC) technique, whereas the remaining (i.e. $y$ and $z$ ) directions are assumed to be periodic. The computational domain is taken to be a cube of $L_{x}=L_{y}=L_{z}=30 \delta_{s t}$ where $\delta_{s t}=$ $\left(T_{a d\left(\phi_{g}=1\right)}-T_{0}\right) / \max |\nabla T|_{L}$ is the thermal flame thickness of the unstrained stoichiometric laminar premixed flame. The simulation domain is discretised by a uniform Cartesian grid of $384^{3}$, which ensures about 10 grid points within $\delta_{s t}$. A commercial software COSILAB (Rotexo-Softpredict-Cosilab) is used to initialise the reacting flow field for three different initial values of droplet diameter $a_{d}$ (i.e. $a_{d} / \delta_{s t}=0.04,0.05$ and 0.06 ) for an overall equivalence ratio $\phi_{o v}$ of unity (i.e. $\phi_{o v}=1.0$ ). The V-flame is subjected to a moderate level of inlet turbulence (i.e. $u^{\prime} / S_{b, s t}=2.0$ ), with a mean inlet velocity of $u_{\text {mean }} / S_{b, s t}=5.0$ and a 
normalised longitudinal integral length scale of $l_{t} / \delta_{s t}=2.5$. The turbulent Reynolds number $R e_{t}=\rho_{0} u^{\prime} L_{t} / \mu_{0}$ (where $L_{t}=k^{1.5} / \varepsilon$ is the integral length scale based on turbulent kinetic energy $k$ and its dissipation rate $\varepsilon$ ) for the flames considered here is about 35.0. It is admitted that the value of $R e_{t}$ remains small but comparable to experiments by Vena et al. $(2011,2015)$ (where $u^{\prime}$ at the inlet varied from $0.19 \mathrm{~m} / \mathrm{s}$ to $0.33 \mathrm{~m} / \mathrm{s}$, the hydraulic diameter of the burner exit is $0.024 \mathrm{~m}$, the integral length scale can be estimated as $7 \%$ of the hydraulic diameter, and the kinematic viscosity is given by $1.8 \times 10^{-5} \mathrm{~m}^{2} / \mathrm{s}$ ). Moreover, the turbulent Reynolds number $R e_{t}$ value of these simulations remains comparable to turbulent premixed V-flame simulations of Dunstan et al. (2011,2012). However, these V-shaped spray flame simulations are considerably more expensive than turbulent premixed V-flame DNS (Dunstan et al., 2011, 2012) because individual droplets are tracked in addition to accounting for gaseous phase combustion. A small value of $u^{\prime} / S_{b, s t}$ is considered in this study because the analysis of the flame-droplet interaction is one of the focal points of this paper and the flame-droplet interaction (e.g. curvature and strain effects) is eclipsed by flame-turbulence interaction for large values of $u^{\prime} / S_{b, s t}$ (Hayashi et al., 1977; Lawes and Saat, 2011; Ozel-Erol et al., 2018,2019).

Table 2. Simulation parameters considered in the analysis.

\begin{tabular}{|c|c|}
\hline Initial droplet diameter, $a_{d} / \delta_{s t}$ & $0.04,0.05,0.06$ \\
\hline Overall Equivalence ratio, $\phi_{o v}$ & 1.0 \\
\hline Heat release parameter, $\tau=\left(T_{a d\left(\phi_{g}=1\right)}-T_{0}\right) / T_{0}$ & 6.54 \\
\hline Unburned gas temperature at the inlet, $T_{0}$ & $300 \mathrm{~K}$ \\
\hline Mean velocity at the inlet, $u_{\text {mean }} / S_{b, s t}$ & 5.0 \\
\hline Inlet turbulence, $u^{\prime} / S_{b, s t}$ & 2.0 \\
\hline Integral length scale, $l_{t} / \delta_{s t}$ & 2.5 \\
\hline Prandtl number $\operatorname{Pr}=\mu C_{p}^{g} / \lambda$ & 0.7 \\
\hline Lewis number, Le & 1.0 \\
\hline Ratio of specific heats $\gamma=C_{p}^{g} / C_{v}^{g}$ & 1.4 \\
\hline Stokes number, $S t$ & 0.13 \\
\hline
\end{tabular}


The steady state laminar spray flame simulation using the methodology adopted by Neophytou and Mastorakos (2009) has been used for obtaining reacting scalar fields under laminar V-flame conditions, which is used as initial conditions for turbulent flame simulations. A plane scans through a frozen field of homogeneous isotropic turbulence to specify turbulent velocity fluctuations at the inlet plane with the help of Taylor's hypothesis. Interested readers are referred to Chakraborty and Cant $(2004)$ and Dunstan et al. $(2011,2012)$ for further discussion on inlet boundary condition specification. The flame holder is placed at approximately $10 \delta_{s t}$ from the inlet plane. The radius of the flame holder is $0.3 \delta_{s t}$ and the statistics are presented at three different axial distances (i.e. position A, B and C) from the flame holder (i.e. $9 \delta_{s t}, 13 \delta_{s t}$ and $17 \delta_{s t}$ ) after $2.0 t_{\text {flow }}$ where $t_{\text {flow }}=L_{x} / u_{\text {mean }}$ is the flow through time. The reactant mass fractions (i.e. $Y_{F}$ and $Y_{O}$ ) and the non-dimensional temperature of gaseous phase $T$ are imposed using a Gaussian weighting function $g(r)=A \exp \left[-r^{2} / 2 \varsigma^{2}\right]$ at the flame holder following Dunstan et al. $(2011,2012)$ where $A$ and $\varsigma$ are adjustable constants to obtain the desired flame holder radius (i.e. $r=0.3 \delta_{s t}$ ). The important simulation parameters are summarised in Table 2.

The initial droplet number density $\rho_{N}$ varies between $1.23 \leq\left(\rho_{N}\right)^{1 / 3} \delta_{t h} \leq 1.85$ in the unburned gas and the liquid volume fraction remains well below 0.01 . The point-source assumption, which is widely used for DNS of diluted spray flames (Reveillon and Vervisch, 2005; Fujita et al., 2013; Neophytou et al., 2012, 2010; Schroll et al., 2009; Wandel, 2014; Wandel et al., 2009; Wang and Rutland, 2005) is employed here to evaluate the position, mass, velocity and temperature of individual droplets in a Lagrangian sense. By contrast, the conservation laws for the gas phase conservation equations are treated in the Eulerian frame of reference. The point source assumption is applicable for droplets, which are smaller than the Kolmogorov length scale and the grid size in DNS. However, these resolution requirements 
restrict this approach for large droplets. The ratio of initial droplet diameter to the Kolmogorov length scale is $a_{d} / \eta=0.09,0.11,0.13$ for the cases of $a_{d} / \delta_{s t}=0.04,0.05,0.06$ respectively for the inlet turbulence intensity (i.e. $u^{\prime} / S_{b, s t}=2.0$ ) considered here. The ratio of the initial droplet diameter to grid spacing used here remains comparable to several previous analyses (Wang and Rutland, 2005; Sreedhara and Huh, 2007; Fujita et al., 2013; Schroll et al., 2009; Wandel et al., 2009; Wandel, 2014; Neophytou et al., 2010, 2012). Furthermore, the diameter of the droplets interacting with the flame in the reaction zone (i.e. the most reacting place in the flame) has been found to be at least $50 \%$ smaller than the initial size due to the relatively high volatility of n-heptane. It is worth noting that Eulerian-Eulerian approaches, which can resolve both gaseous and liquid phases, also have some limitations (i.e. to capture the polydispersity and droplet crossing). Moreover, fully resolved dispersed phase simulations (Eulerian-Eulerian methods) for reacting flows are still not in a mature state and need further validation. As a reference, de Chaisemartin et al. (2009) compared the combustion of polydisperse droplets in a two-dimensional free jet using DNS with point source assumption and fully Eulerian methodologies. It was indicated that the Eulerian description of droplets yields a smoother gaseous mass fraction field in comparison to the Lagrangian description. However, the simulations with fully Eulerian approach were reported 10 times more expensive than those with point source assumption and it may require a huge parallelisation capability for 3D simulations. The Stokes number $S t=\tau_{p} \sqrt{k} / l_{t}=\rho_{d} a_{d}^{2} \sqrt{k} /\left(18 C_{u} \mu l_{t}\right) \quad\left(\right.$ where $\tau_{p}=$ $\rho_{d} a_{d}^{2} / 18 C_{u} \mu$ is the particle time scale and $l_{t} / \sqrt{k}$ is the turbulent time scale) remains smaller than 0.13 for the cases considered here. Alternatively, the Stokes number $S t^{\prime}=\tau_{p} S_{b, s t}^{2} / \alpha_{T 0}=$ $\rho_{d} a_{d}^{2} S_{b, s t}^{2} /\left(18 C_{u} \mu \alpha_{T 0}\right)$ can be estimated based on the chemical time scale $\alpha_{T 0} / S_{b, s t}^{2}$ and the maximum value of $S t^{\prime}$ remains smaller than 0.27 for the largest droplets considered in this analysis. The mean normalised inter-droplet distance $s_{d} / \eta$ ranges between 1.64 and 2.47 for 
the inlet turbulence intensity considered here.

A group number, $\mathrm{G}=3\left(1+0.276 R e_{d}^{1 / 2} S c^{1 / 3}\right) L e N^{2 / 3}\left(a_{d} / s_{d}\right)$ (where Le and $S c$ are the Lewis and Schmidt numbers, respectively, $s_{d}$ is the distance between droplets, $N$ is the number of droplets in a specified volume) can be defined following Chiu and Liu (1977) in order to distinguish between individually burning droplets $(\mathrm{G} \ll 1.0)$ and external sheath combustion $(G \gg 1.0)$. All droplet cases considered in the current study come under the category of external sheath combustion (i.e. G remains much greater than unity). In the case of the small value of G pyrolysis takes place at the fuel-rich core due to extremely high temperature, whereas the temperature values in the external sheath combustion are usually not high enough to give rise to a significant amount of pyrolysis (Kuo and Acharya, 2012). For the present configuration, the burned gas temperature remains either comparable or smaller than the adiabatic flame temperature of the stoichiometric mixture, and thus the effects of pyrolysis are expected to be minimal. Moreover, thermochemistry for the present analysis involves a modified single-step Arrhenius-type chemical mechanism for the computational economy, and this chemical mechanism is not adequate for the analysis of the pyrolysis process. A similar assumption was made in several previous analyses (Wang and Rutland, 2005; Sreedhara and Huh, 2007; Fujita et al., 2013; Schroll et al., 2009; Wandel et al., 2009; Wandel, 2014; Neophytou et al., 2010, 2012) and the same approach has been adopted here. A recent DNS analysis by Zhou et al. (2019) demonstrated with the help of the Kerstein-Law parameter that either group combustion or individual burning is unlikely to occur under conditions which are comparable to the conditions analysed here. 

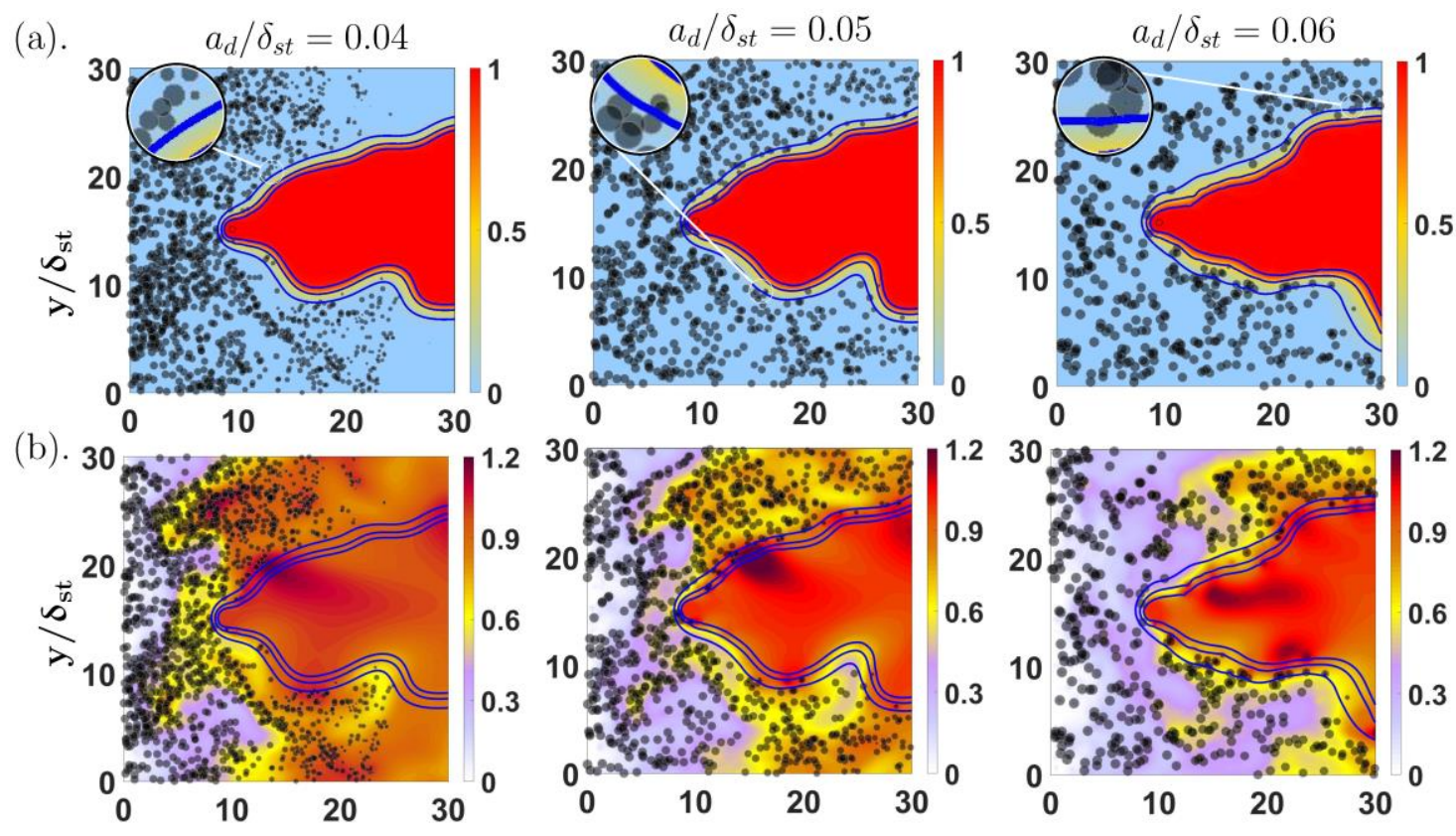

(c).
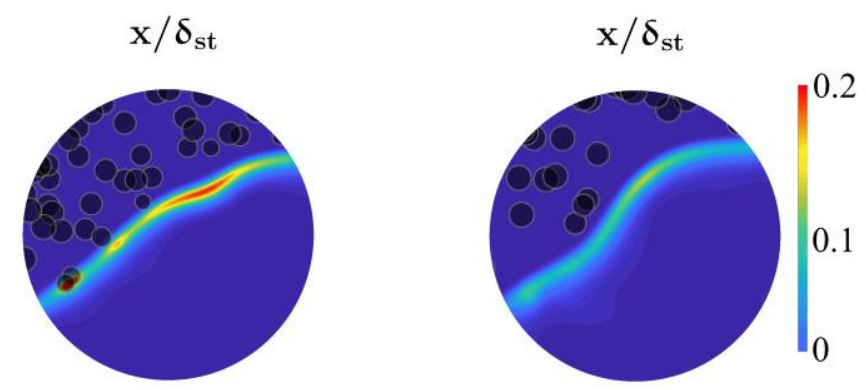

Figure 2. Instantaneous distributions of (a) reaction progress variable, $c$ (b) gaseous equivalence ratio, $\phi_{g}$ and (c) fuel reaction rate magnitude $\left|\dot{w}_{F}\right| \times \delta_{s t} / \rho_{0} S_{b\left(\phi_{g}=1\right)}$ on the central x-y midplane for initial $a_{d} / \delta_{s t}=0.04,0.05,0.06$ at $t=2.0 t_{\text {flow }}$. Blue lines show $c$ $=0.1,0.5$ and 0.9 contours from outer to inner periphery and grey dots show the droplets residing on the plane (not to the scale).

\section{RESULTS AND DISCUSSION}

The contours of reaction progress variable, $c$, gaseous equivalence ratio, $\phi_{g}$ and fuel reaction rate magnitude $\left|\dot{w}_{F}\right| \times \delta_{s t} / \rho_{0} S_{b\left(\phi_{g}=1\right)}$ on the central x-y mid-plane at $t=2.0 t_{\text {flow }}$ are shown in Fig. 2 for initial droplet diameters $a_{d} / \delta_{s t}=0.04,0.05,0.06$. The droplets residing on the plane are illustrated by grey dots. It can be seen from the reaction progress variable distribution shown in Fig. 2 that the background fluid turbulence creates wrinkling on the flame surface. It is worth noting that fuel is supplied in the form of liquid droplets at the inlet which is placed on the left-hand side of the domain. Liquid fuel droplets, distributed randomly but statistically 
uniform in space at the inlet plane, are introduced with a temperature of $T_{0}=300 \mathrm{~K}$. The mass flux of liquid fuel at the inlet plane is estimated based on the values of $u_{\text {mean }}$ and $\phi_{o v}$. These droplets shrink in size due to evaporation as they approach the flame (see insets in Fig. 2) and the combustion of the evaporated gaseous fuel sustains the V-shaped flame. It can be seen from Fig. 2 that small droplets with initial $a_{d} / \delta_{s t}=0.04$ mostly complete their evaporation by halfway in the streamwise direction due to their high evaporation rate, whereas large droplets with initial $a_{d} / \delta_{s t}=0.05$ and 0.06 survive longer due to their lower evaporation rate and interact more with the flame than smaller droplets. Although an overall stoichiometric fuel-air mixture is provided in the unburned gas (note that fuel in the gaseous phase is absent at the inlet), the evaporation ahead of the flame is not sufficient to produce a gaseous stoichiometric mixture (i.e. $\phi_{g}=1.0$ ) and this tendency is particularly prevalent for the case with initial $a_{d} / \delta_{s t}=0.06$. Although the gaseous phase combustion in V-flames takes place under $\phi_{g}<1.0$ for all droplet cases, the likelihood of finding fuel-air mixture corresponding to $\phi_{g} \approx$ 1.0 is comparatively higher for the initial $a_{d} / \delta_{s t}=0.04$ case. This behaviour arises due to the faster evaporation of smaller droplets. Some of the droplets penetrate the flame structure in the $a_{d} / \delta_{s t}=0.06$ case and they eventually evaporate in the reaction zone and in the burned gas to produce local islands of fuel-rich (i.e. $\phi_{g}>1.0$ ) mixtures. It is worth noting that the evaporation of droplets leads to higher availability of fuel vapour, which in turn gives rise to local islands of high reaction rate magnitude $\left|\dot{w}_{F}\right| \times \delta_{s t} / \rho_{0} S_{b\left(\phi_{g}=1\right)}$ as can be seen from Fig. $2 \mathrm{c}$ where the magnified views of the regions with $\phi_{g} \approx 1.0$ are shown. The latent heat of evaporation $L_{v}$ affects the temporal evolution of droplet temperature $d T_{d} / d t$ through the Spalding number $B_{d}$. Most droplets complete their evaporation process in the reaction zone and thus the latent heat of evaporation acts to reduce the temperature and reaction rate magnitude but the availability of the fuel vapour due to evaporation tends to increase reaction rate 
magnitude. The relative strengths of these two competing effects determine the net effect of the latent heat on the reaction zone. However, the extraction of the latent heat reduces the temperature of the gas within the preheat zone which acts to reduce the most probable burned gas temperature in comparison to the adiabatic flame temperature of the stoichiometric mixture. This was discussed earlier by Nakamura et al. (2005) and Wacks et al. (2016) and the qualitatively similar behaviour has been observed in the flames considered here and thus are not explicitly shown here.
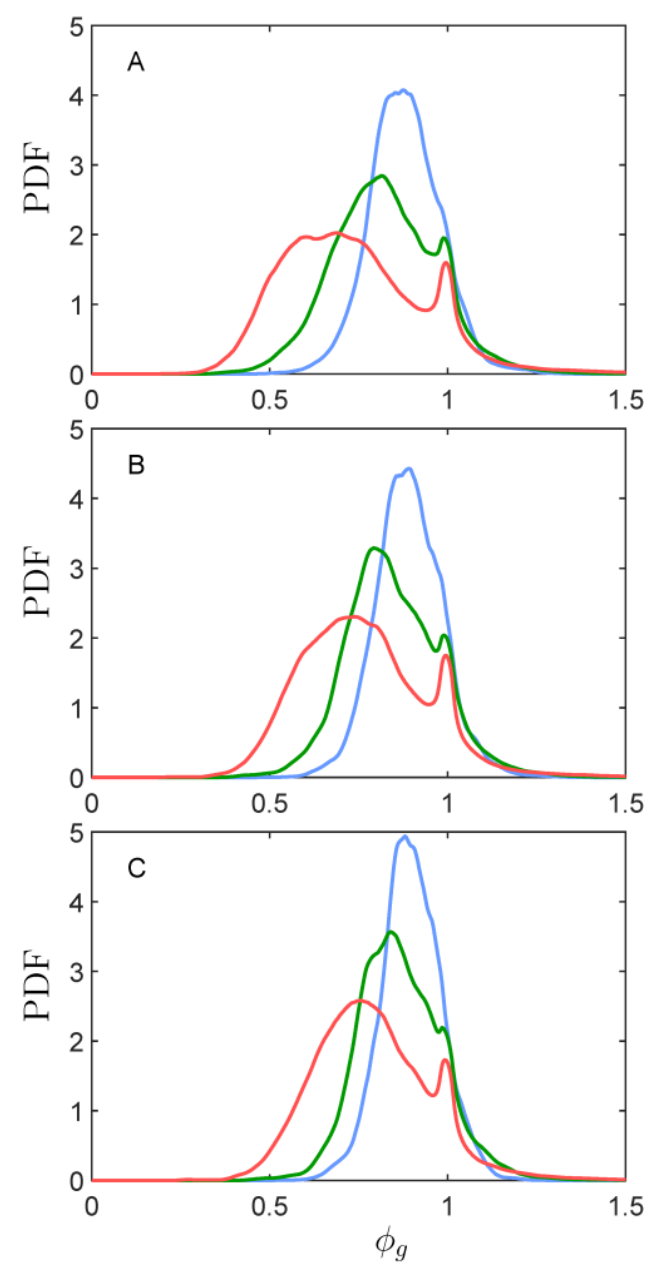

Figure 3. PDFs of $\phi_{g}$ in the region corresponding to $0.01 \leq c \leq 0.99$ at different locations

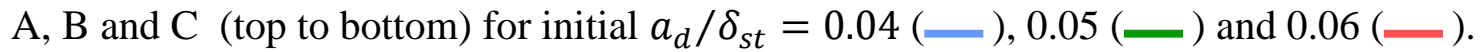

In order to illustrate the composition of the gaseous phase mixture within the flame, the 
probability density functions (PDFs) of the gaseous phase equivalence ratio $\phi_{g}$ within the region given by $0.01 \leq c \leq 0.99$ at three different axial locations (i.e. at distances $x / \delta_{s t}=9$, 13 and 17 from the flame holder, which are indicated as locations A, B and C, respectively) are presented in Fig. 3. It is evident from Fig. 3 that combustion predominantly takes place under fuel-lean mode for all locations and droplet diameters. However, the cases with small droplets (i.e. initial $a_{d} / \delta_{s t}=0.04$ and 0.05 cases) show a considerable likelihood of finding $\phi_{g} \approx 1.0$, especially at locations B and C. Furthermore, the PDF of $\phi_{g}$ for large droplets with initial $a_{d} / \delta_{s t}=0.06$ shows a mild peak at $\phi_{g}=1.0$ at all locations but the probability of obtaining $\phi_{g}<1.0$ remains greater than that of finding $\phi_{g} \geq 1.0$ in all cases. The probability of having a fuel-lean mode of combustion decreases in the downstream direction due to increased availability of fuel vapour as a result of evaporation of droplets. For example, the droplet number density $\left(\rho_{N}\right)^{1 / 3} \delta_{t h}$ in the region corresponding to $0.01 \leq c \leq 0.99$ drops by $30 \%, 4 \%$ and $1.14 \%$ at location $\mathrm{C}$ in comparison to location $\mathrm{A}$ for cases with initial $a_{d} / \delta_{s t}=0.04,0.05$ and 0.06 , respectively. Therefore, the flame at plane $\mathrm{A}$ is leaner than in plane $\mathrm{C}$, which can be substantiated from the fact that the time-averaged values of $\phi_{g}$ for $a_{d} / \delta_{s t}=0.04$ are 0.88 , 0.89 and 0.90 , for $a_{d} / \delta_{s t}=0.05$ are $0.83,0.85$ and 0.87 , for $a_{d} / \delta_{s t}=0.06$ are $0.74,0.77$ and 0.79, at locations A, B and C, respectively. It is worth noting that the PDFs of $\phi_{g}$ in Fig. 3 are shown only for $0.01 \leq c \leq 0.99$ and a comparison between Figs. $2 \mathrm{a}$ and $2 \mathrm{~b}$ reveals that it is possible that there are local islands of highly fuel-rich mixtures in the fully burned regions because of the mixing of evaporated vapour from the neighborhood and also due to small number of droplets which escape through the flame due to turbulent motion and complete the evaporation in the high temperature zone in the region with $c=1.0$. Some of these unburned fuel-pockets eventually diffuse into the flame and mix with the air diffusing from the unburned gas side to give rise to local diffusion mode of burning, which will be discussed later in this 
paper. Moreover, a close inspection of Fig. 3 reveals that the peak value of the PDF of $\phi_{g}$ at location $\mathrm{C}$ is found close to $\phi_{g}=1.0$ (i.e. most probable value of $\phi_{g}$ is going to be close to 1.0). This clearly indicates that the PDF of $\phi_{g}$ within the flame is likely to see a peak at $\phi_{g}=$ 1.0 and a mean value of $\phi_{g}$ close to unity in the case of $a_{d} / \delta_{s t}=0.04$ at a location which is further away from the flame holder than the location $\mathrm{C}$. This behaviour can also be discerned from the distributions of $\phi_{g}$ in Fig. 2b. It is worth noting that the fuel-lean nature of the gaseous reacting mixture is qualitatively consistent with previous findings for laminar flame calculations (Neophytou and Mastorakos, 2009), statistically planar turbulent spray flames (Wacks et al, 2016) and spherically expanding turbulent spray flames Ozel-Erol et al. (2018). Thus, this fuel-lean nature of the gaseous phase combustion for globally stoichiometric (i.e. $\left.\phi_{o v}=1.0\right)$ spray flames has been found to be mostly independent of the flame configuration but is influenced by the liquid droplet size, turbulence intensity and number density of liquid droplets (Wacks et al., 2016; Ozel-Erol et al., 2018). However, droplets interact with both statistically planar and spherically expanding flames at all stages of their propagation into the unburned gas but in turbulent V-spray flames, droplets travel in the mean flow direction during the evaporation process. As a result, the frequency of flame-droplet interaction decreases and the mean value of $\phi_{g}$ increases in the downstream direction.

It is worth noting that droplets do not evaporate instantly and the evaporated fuel vapour does not immediately homogeneously mix to produce a mixture with $\phi_{g}=1.0$ even when the overall equivalence ratio $\phi_{o v}$ takes a value of 1.0. Thus the limiting case for small diameter is a gaseous stratified mixture flame but not a premixed flame, but this stratification cannot be a priori characterised. It has been shown in previous analyses on stratified combustion (Hélie and Trouvé, 1998; Malkeson and Chakraborty, 2010) that the burning rate and flame front thickness 
for stratified mixture with a mean equivalence ratio of $\phi_{g}=1.0$ can be smaller than in the stoichiometric premixed flames due to smaller burning rates of $\phi_{g}<1.0$ and $\phi_{g}>1.0$ mixtures.

Table 3:Variation of the mean droplet diameter normalised by its initial value $a_{d, m} / a_{d}$ in the unburned gas region at locations $\mathrm{A}, \mathrm{B}, \mathrm{C}$.

\begin{tabular}{|c|c|c|c|c|}
\hline & & $a_{d} / \delta_{s t}=0.04$ & $a_{d} / \delta_{s t}=0.05$ & $a_{d} / \delta_{s t}=0.06$ \\
\hline \multirow{4}{*}{$\varangle$} & $0 \leq c<0.1$ & 0.36 & 0.60 & 0.74 \\
\cline { 2 - 5 } & $0.1 \leq c<0.3$ & 0.25 & 0.45 & 0.64 \\
\cline { 2 - 5 } & $0.3 \leq c<0.5$ & Absent & 0.33 & 0.54 \\
\hline \multirow{4}{*}{} & $0 \leq c<0.1$ & 0.29 & 0.54 & 0.70 \\
\cline { 2 - 5 } & $0.1 \leq c<0.3$ & 0.20 & 0.39 & 0.59 \\
\cline { 2 - 5 } & $0.3 \leq c<0.5$ & Absent & 0.29 & 0.49 \\
\hline \multirow{4}{*}{$\circlearrowright$} & $0 \leq c<0.1$ & 0.23 & 0.49 & 0.67 \\
\cline { 2 - 5 } & $0.1 \leq c<0.3$ & 0.15 & 0.32 & 0.54 \\
\cline { 2 - 5 } & $0.3 \leq c<0.5$ & Absent & 0.27 & 0.43 \\
\hline
\end{tabular}

The mean droplet diameters for $0 \leq c<0.1,0.1<c \leq 0.3$ and $0.3<c \leq 0.5$ at locations $\mathrm{A}$, $\mathrm{B}$ and $\mathrm{C}$ are shown in Table 3. It is worth noting that only few droplets survive for $c>0.5$ so the statistical average based on limited samples are not meaningful. It can be seen from Table 3 that the mean droplet size decreases due to evaporation as $c$ increases but the differences in mean diameter size increase within the flame because smaller droplets evaporate faster. It can also be seen from Table 3 that the mean droplet size in the region corresponding to $0 \leq c<0.1$ remains comparable to the initial values of $a_{d} / \delta_{s t}$ for large droplets, whereas smaller droplets are mostly evaporated when they reach location A as shown in Fig. 2. The evaporation process before reaching the location A leads to significant diameter differences between the cases with different droplet diameters in the V-flame configuration. Therefore, the mean droplet sizes in the zones corresponding to $0 \leq c<0.1,0.1<c \leq 0.3$ and $0.3<c \leq 0.5$ consist of a wide spread of droplet diameter at location A due to high (low) evaporation rate of small (large) droplets. It would not be appropriate to compare the mean droplet diameter at location A, B 
and $\mathrm{C}$ in the V-flames with spherically expanding flames. However, it is worth noting that the mean droplet sizes in the zones corresponding to $0 \leq c<0.1,0.1<c \leq 0.3$ and $0.3<c \leq$ 0.5 in the spherically expanding flame with $\phi_{o v}=1.0$ by the present authors (Ozel-Erol et al., 2018) show the the same qualitative trend to the V-flame configuration, especially for the large droplet case.

The Damköhler and Karlovitz numbers for the stoichiometric gaseous mixture based on the inlet values of $u^{\prime} / S_{b, s t}$ and $l_{t} / \delta_{s t}$ are 1.25 and 1.79 (i.e. $D a_{\left(\phi_{g}=1\right)}=l_{t} S_{b, s t} / u^{\prime} \delta_{s t}=1.25$ and $\left.K a_{\left(\phi_{g}=1\right)}=\left(u^{\prime} / S_{b, s t}\right)^{1.5}\left(l_{t} / \delta_{s t}\right)^{-0.5}=1.79\right)$, respectively. The predominant likelihood of fuel-lean combustion in these droplet V-flame cases is expected to increase the Karlovitz number and decrease the Damköhler number of the underlying combustion process in comparison to $D a_{\left(\phi_{g}=1\right)}$ and $K a_{\left(\phi_{g}=1\right)}$, respectively. The local Damköhler number can alternatively be estimated by $D a_{L}=L_{t u r b} S_{b, \tilde{\xi}}^{2} / u_{r m s} \alpha_{T_{0}}$ with $L_{t u r b}=\tilde{k}^{1.5} / \tilde{\varepsilon}$ and $u_{r m s}=$ $\sqrt{2 \tilde{k} / 3}$ being local turbulent length scale and root-mean-square velocity with $\tilde{k}$ and $\tilde{\varepsilon}$ being the Favre-averaged turbulent kinetic energy and its dissipation rate, respectively. Here $S_{b, \tilde{\xi}}$ is the laminar burning velocity corresponding to $\phi_{R}=\tilde{\xi}\left(1-\xi_{s t}\right) /\left[\xi_{s t}(1-\tilde{\xi})\right]$. The local values of the Damköhler number are exemplarily provided at the location corresponding to $\tilde{c}=0.5$, which for $a_{d} / \delta_{s t}=0.04$ are $0.79,0.92$ and 1.09 , for $a_{d} / \delta_{s t}=0.05$ are $0.49,0.71$ and 1.00, for $a_{d} / \delta_{s t}=0.06$ are $0.27,0.40$ and 0.62 , for the premixed case are $3.66,3.93,3.99$ at locations A, B and C, respectively. Similarly, the corresponding values of the local Karlovitz number $\left(K a_{L}=\left(u_{r m s} / S_{b, \tilde{\xi}}\right)^{1.5}\left(L_{t u r b} S_{b, \tilde{\xi}} / \alpha_{T_{0}}\right)^{-0.5}\right)$ based on $\tilde{k}, \tilde{\varepsilon}$ and $S_{b, \tilde{\xi}}$ values at the location corresponding to $\tilde{c}=0.5$ for $a_{d} / \delta_{s t}=0.04$ are $1.79,1.74$ and 1.79 , for $a_{d} / \delta_{s t}=$ 0.05 are $2.60,2.18$ and 1.87 , for $a_{d} / \delta_{s t}=0.06$ are $4.12,3.33$ and 2.75 , for the premixed case 
are 3.77, 3.39, 2.91 at locations A, B and C, respectively. According to these estimated values of $D a_{L}$ and $K a_{L}$, combustion takes places in the thin reaction zones regime (Peters, 2000). The expression "thin reaction zones regime" refers to a condition where the reaction zone thickness remains smaller than the Kolmogorov length scale and thus the reaction processes remain quasilaminar. In this sense, the reaction occurs in a thin region in comparison to the dissipative length scale of turbulence. The differences in $D a_{L}$ and $K a_{L}$ values between gaseous premixed and droplet flames arise due to the differences in respective $S_{b, \tilde{\xi}}, \tilde{k}$ and $\tilde{\varepsilon}$ values originating from mixture composition and also due to difference in turbulence evolution within the flame front. This behaviour can be further illustrated by the PDFs of reaction progress variable $c$ on the isosurfaces of the Favre-averaged reaction progress variable $\tilde{c}=0.1,0.5$ and 0.9 at locations A, B and C, which are shown in Fig. 4, whereas the contours of $\tilde{c}=0.1,0.5$ and 0.9 for the cases considered here are shown in Fig. 5a. In this paper, the Favre averaging is carried out by averaging in time (i.e. over flow through time $t_{\text {flow }}$ ) and space (i.e. spanwise zdirection). For high values of Damköhler number (i.e. $D a_{\left(\phi_{g}\right)} \gg 1$ ), the PDFs of $c$ are expected to be bimodal with impulses at $c=0.0$ and $c=1.0$ (Bray et al., 1980) but Fig. 4 shows that the PDFs of $c$ do not remain bimodal (in fact the PDFs are nearly monomodal in some instances for the cases considered here) and the probability of finding reacting mixture (i.e. $0.0<c<$ 1.0) remains significant for all locations irrespective of the value of $\tilde{c}$. The departure of the PDFs of $c$ from the bimodal distribution with impulses at $c=0.0$ and $c=1.0$ provides the signature of low Damköhler number combustion (i.e. $D a_{\left(\phi_{g}\right)}<1$ ) for the cases considered here. This suggests that combustion in these V-shaped flames takes place in the thickened flame regime and this behaviour is prominent for the case with initial $a_{d} / \delta_{s t}=0.06$, where local occurrences of flame thickening can be discerned from Fig. 2. Moreover, the appearance of the morphology of these V-flame surfaces has been found to be qualitatively similar to the 
experimental visualisations of premixed V-flames in the thickened flame regime (Kheirkhah and Gulder, 2014). The turbulent Reynolds number remains moderate for all cases considered here. Moreover, turbulence decays within the flame and kinematic viscosity increases with increasing $c$ as a result of temperature rise. This clearly suggests that the flame is weakly turbulent, and the effects of intermittency are expected to be prevalent in these flames. As a consequence, the internal flame structure remains quasi-laminar in nature but the combination of $D a<1$ and weak turbulence suggests that the local occurrences of flame-front thickening takes place due to penetration of occasional turbulent eddies within the preheat zone and thus the preheat zone is perturbed, but the reaction zone remains quasi-laminar. This is consistent with the aforementioned Karlovitz number values suggesting the thin reaction zones regime combustion (Peters, 2000) in the cases considered here.
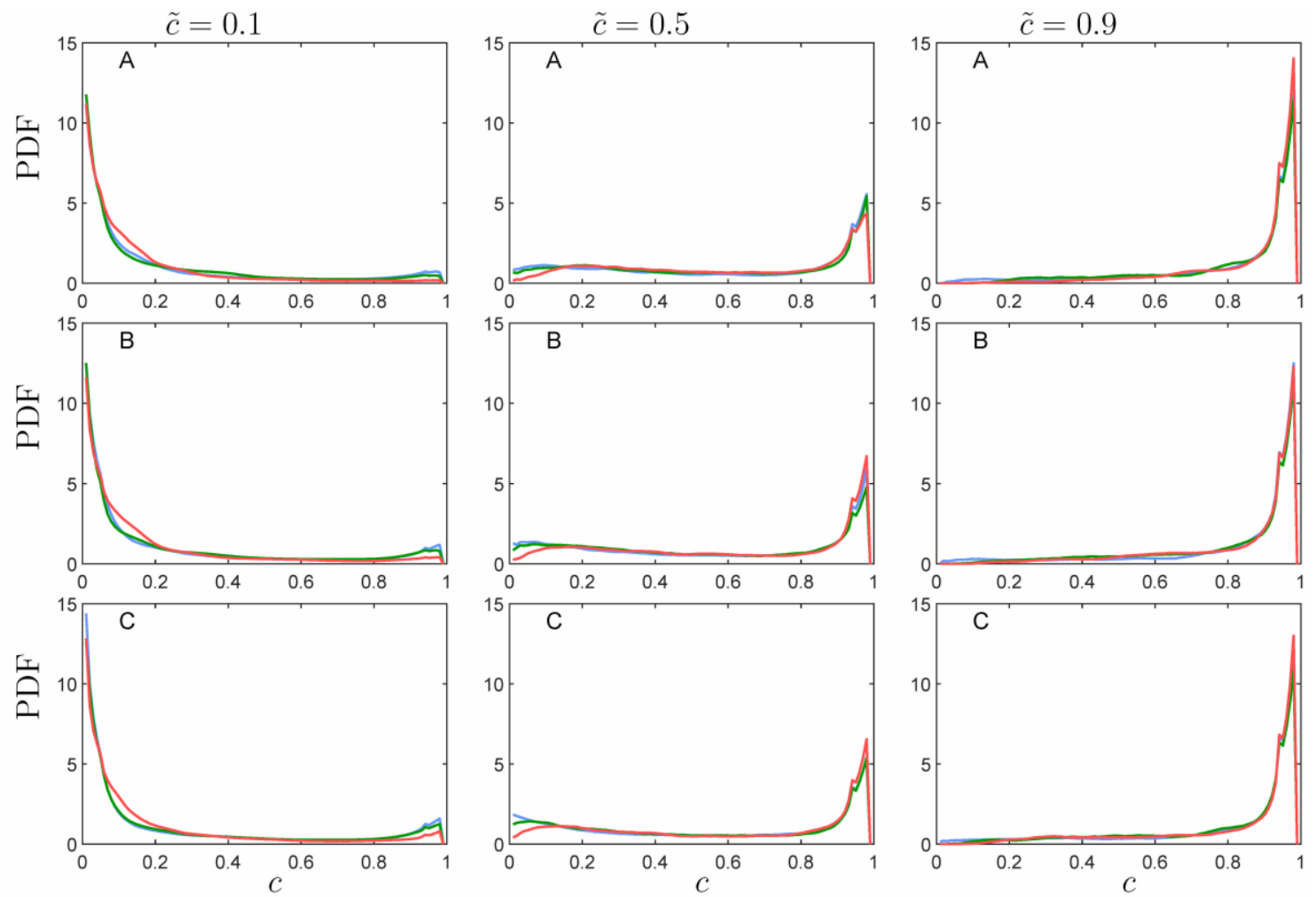

Figure 4. PDFs of $c$ conditional on $\tilde{c}=0.1,0.5,0.9$ at different locations A, B and C (top to bottom) for initial $a_{d} / \delta_{s t}=0.04(-), 0.05$ (_) and $0.06\left(\_\right)$. 
It can be seen from Fig. 5 that the distance between $\tilde{c}=0.1$ and 0.9 isosurfaces (i.e. $\delta_{T(0.1-0.9)}$ ) at a given $x$-location increases in the downstream direction from the flame holder, which is consistent with turbulent diffusion theory (Langevin, 1908; Taylor, 1922; Peters, 2000; Pope, 2000). This behaviour is qualitatively similar to turbulent premixed V-flame simulations (Kheirkhah and Gulder, 2014; Dunstan et al., 2011, 2012). The flame brush thickness $\delta_{T, \tilde{c}}$ at a given location can be quantified as:

$$
\delta_{T, \tilde{c}}=1 / \max |\nabla \tilde{c}|
$$

Alternatively, a flame brush thickness $\delta_{T, \bar{c}}$ can be defined as:

$$
\delta_{T, \bar{c}}=1 / \max |\nabla \bar{c}|
$$

Table 4. Flame brush thickness at locations A, B and C

\begin{tabular}{|c|c|c|c|c|}
\hline Case & $\begin{array}{c}\text { Gaseous } \\
\text { premixed } \\
\text { flame }\end{array}$ & $\frac{\boldsymbol{a}_{\boldsymbol{d}}}{\boldsymbol{\delta}_{\boldsymbol{s t}}}=\mathbf{0 . 0 4}$ & $\frac{\boldsymbol{a}_{\boldsymbol{d}}}{\boldsymbol{\delta}_{\boldsymbol{s t}}}=\mathbf{0 . 0 5}$ & $\frac{\boldsymbol{a}_{\boldsymbol{d}}}{\boldsymbol{\delta}_{\boldsymbol{s t}}}=\mathbf{0 . 0 6}$ \\
\hline$\delta_{T(0.1-0.9)} / \delta_{s t}$ at A & 3.14 & 3.45 & 3.38 & 3.23 \\
\hline$\delta_{T(0.1-0.9)} / \delta_{s t}$ at B & 3.34 & 4.13 & 4.00 & 3.75 \\
\hline$\delta_{T(0.1-0.9)} / \delta_{s t}$ at C & 3.70 & 4.50 & 4.40 & 4.06 \\
\hline$\delta_{T, \bar{c}} / \delta_{s t}$ at A & 3.04 & 3.50 & 3.33 & 3.18 \\
\hline$\delta_{T, \bar{c}} / \delta_{s t}$ at B & 3.12 & 4.27 & 4.12 & 3.83 \\
\hline$\delta_{T, \bar{c}} / \delta_{s t}$ at C & 3.35 & 4.79 & 4.97 & 4.35 \\
\hline$\delta_{T \tilde{c}} / \delta_{s t}$ at A & 2.47 & 2.82 & 2.69 & 2.72 \\
\hline$\delta_{T \tilde{c}} / \delta_{s t}$ at B & 2.34 & 3.28 & 3.12 & 3.09 \\
\hline$\delta_{T \tilde{c}} / \delta_{s t}$ at C & 2.37 & 3.84 & 3.62 & 3.45 \\
\hline
\end{tabular}

The values of $\delta_{T(0.1-0.9)} / \delta_{s t}, \delta_{T, \tilde{c}} / \delta_{s t}$ and $\delta_{T, \bar{c}} / \delta_{s t}$ are reported in Table 4 for locations A, B and C. It can be seen from Table 4 that the values of $\delta_{T, \bar{c}} / \delta_{s t}$ and $\delta_{T(0.1-0.9)} / \delta_{s t}$ for the droplet cases remain close to each other but the value of $\delta_{T, \tilde{c}} / \delta_{s t}$ remains smaller than $\delta_{T, \bar{c}} / \delta_{s t}$ and $\delta_{T(0.1-0.9)} / \delta_{s t}$. A comparison with the corresponding gaseous premixed flame indicates that the flame brush is thicker in droplet cases than in the premixed gaseous case. It has been shown 
in Fig. 3 that spray flames experience considerable amount of fuel-lean (i.e. $\left.\phi_{g}<1.0\right)$ mixture, and the flamelet thickness $\left(\sim \alpha_{T 0} / S_{b\left(\phi_{g}\right)}\right)$ is expected to be greater, whereas laminar burning velocity $S_{b\left(\phi_{g}\right)}$ is smaller than the corresponding values for the stoichiometric premixed flame. Thus, the spray flames experience higher values of turbulence intensity $u^{\prime} / S_{b\left(\phi_{g}\right)}$ and are expected to show greater values of flamelet thickness and a combination of these gives rise to greater values of flame brush thickness in the spray flames than in the stoichiometric premixed flames, which can be substantiated from Table 4. It is worth noting that the evolution of turbulence (i.e. rms turbulent velocity and length scale) within the spray flames for different droplet sizes are likely to be different, which plays a role in determining the flame brush thickness (Meneveau and Poinsot, 1991). For example, the decay of turbulence across the flame is likely to be less rapid for large droplets in comparison to small droplets because the effects of heat release are likely to be weaker due to burning of leaner mixtures. Therefore, the flame brush thickness for spray flames is not dictated only by the droplet diameter but also by the length scale and turbulence intensity within the flame brush, which is beyond the scope of this analysis. It is also worth noting that a premixed flame is not the limiting case of the small droplet diameter so it should not be expected that the premixed flame is going to follow the trend shown by the spray flames.

The change in the flame brush thickness in the flow direction indicates that the angles made by the tangent on the $\tilde{c}=0.1,0.5$ and 0.9 contours with the mean flow direction (i.e. $x$-direction) are expected to be different, which can also be verified from Table 5 where the angles $\theta_{\tilde{c}=0.1}$, $\theta_{\tilde{c}=0.5}$ and $\theta_{\tilde{c}=0.9}$ for $\tilde{c}=0.1,0.5$ and 0.9 isosurfaces are also listed for locations A, B and C. The angles $\theta_{\tilde{c}=0.1}, \theta_{\tilde{c}=0.5}$ and $\theta_{\tilde{c}=0.9}$ are evaluated by using the slope of the tangents at locations A, $\mathrm{B}$ and $\mathrm{C}$ on the contour lines of $\tilde{c}=0.1,0.5$ and 0.9 , as shown in Fig. 5b. 

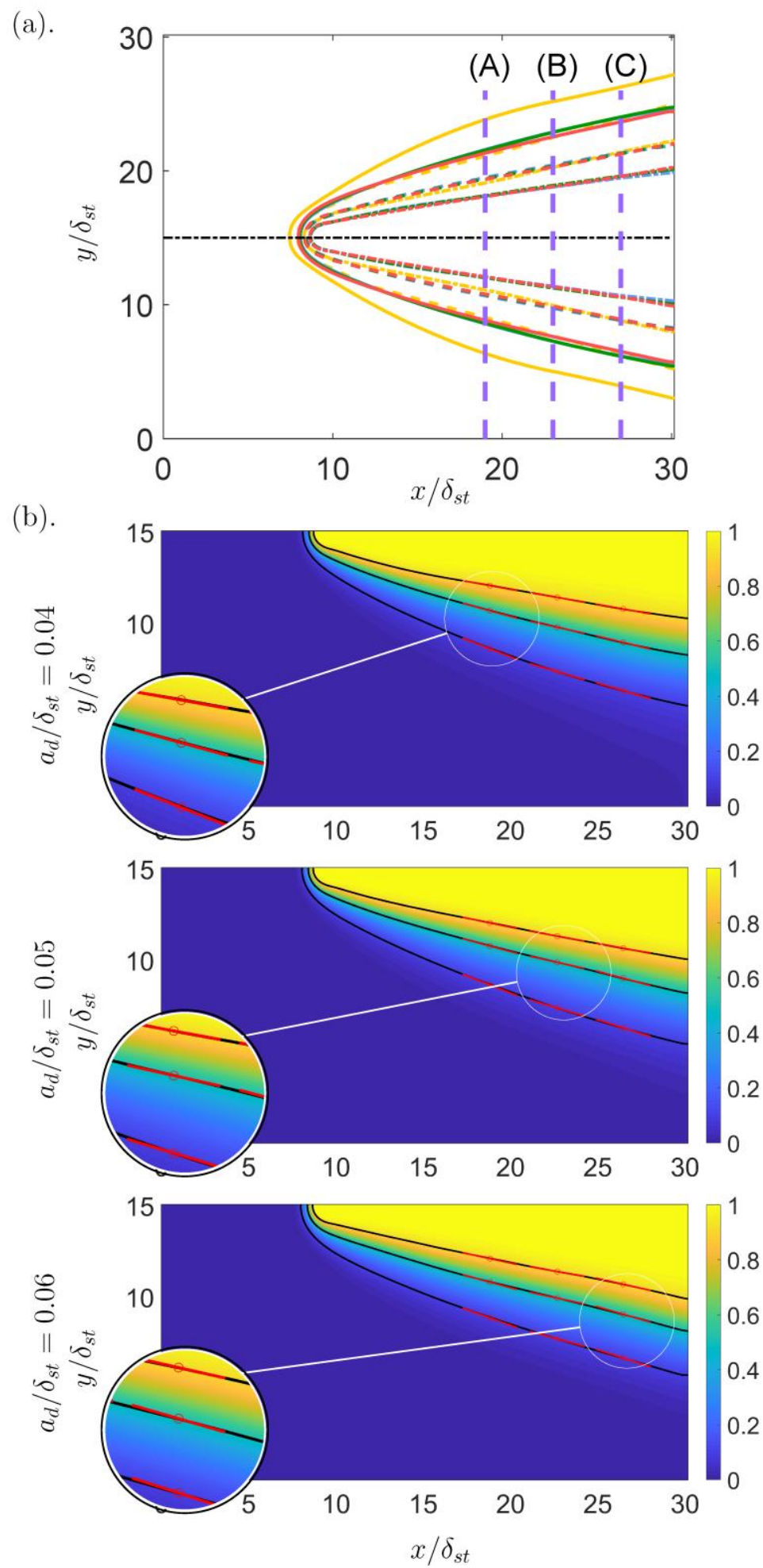

Figure 5. (a) Contours of $\tilde{c}=0.1$ (solid line), 0.5 (dashed line) and 0.9 (dotted line) contours for gaseous premixed ( $\_$) case and droplet cases with initial droplet diameter $a_{d} / \delta_{s t}=$

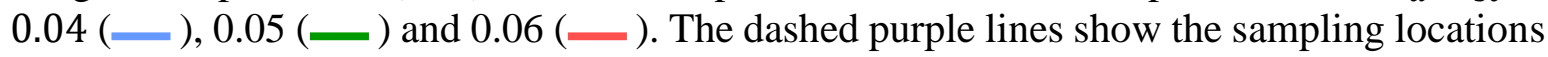
(A, B, C from left to right), and the dotted black line shows the flame centre, (b) Distributions of $\tilde{c}$ at the bottom half of the domain for initial $a_{d} / \delta_{s t}=0.04$ ( $1^{\text {st }}$ row $), 0.05$ ( $2^{\text {nd }}$ row $)$ and 0.06 ( $3^{\text {rd }}$ row). Black lines show the contour lines of $\tilde{c}=0.1,0.5$, and 0.9 from outer to the inner periphery and red lines are tangents to contour lines at locations $\mathrm{A}, \mathrm{B}$ and $\mathrm{C}$ (left to right). 
Table 5. Flame angle $\theta$ for $\tilde{c}$ isosurfaces

\begin{tabular}{|l|c|c|c|c|}
\hline \multicolumn{1}{|c|}{ Case } & $\begin{array}{c}\text { Gaseous } \\
\text { Premixed } \\
\text { Flame }\end{array}$ & $\frac{\boldsymbol{a}_{\boldsymbol{d}}}{\boldsymbol{\delta}_{\boldsymbol{s t}}}=\mathbf{0 . 0 4}$ & $\frac{\boldsymbol{a}_{\boldsymbol{d}}}{\boldsymbol{\delta}_{\boldsymbol{s t}}}=\mathbf{0 . 0 5}$ & $\frac{\boldsymbol{a}_{\boldsymbol{d}}}{\boldsymbol{\delta}_{\boldsymbol{s t}}}=\mathbf{0 . 0 6}$ \\
\hline Flame angle $\theta_{\tilde{c}=0.1}$ for $\tilde{c}=0.1$ at A & $16.06^{\circ}$ & $20.47^{\circ}$ & $20.42^{\circ}$ & $18.88^{\circ}$ \\
\hline Flame angle $\theta_{\tilde{c}=0.1}$ for $\tilde{c}=0.1$ at B & $13.56^{\circ}$ & $15.76^{\circ}$ & $16.12^{\circ}$ & $14.80^{\circ}$ \\
\hline Flame angle $\theta_{\tilde{c}=0.1}$ for $\tilde{c}=0.1$ at $\mathrm{C}$ & $17.12^{\circ}$ & $15.46^{\circ}$ & $16.58^{\circ}$ & $17.00^{\circ}$ \\
\hline Flame angle $\theta_{\tilde{c}=0.5}$ for $\tilde{c}=0.5$ at A & $14.66^{\circ}$ & $14.66^{\circ}$ & $14.49^{\circ}$ & $11.72^{\circ}$ \\
\hline Flame angle $\theta_{\tilde{c}=0.5}$ for $\tilde{c}=0.5$ at B & $7.21^{\circ}$ & $12.07^{\circ}$ & $12.06^{\circ}$ & $15.44^{\circ}$ \\
\hline Flame angle $\theta_{\tilde{c}=0.5}$ for $\tilde{c}=0.5$ at $\mathrm{C}$ & $10.95^{\circ}$ & $13.04^{\circ}$ & $14.37^{\circ}$ & $14.99^{\circ}$ \\
\hline Flame angle $\theta_{\tilde{c}=0.9}$ for $\tilde{c}=0.9$ at A & $13.46^{\circ}$ & $9.82^{\circ}$ & $11.20^{\circ}$ & $11.62^{\circ}$ \\
\hline Flame angle $\theta_{\tilde{c}=0.9}$ for $\tilde{c}=0.9$ at B & $10.57^{\circ}$ & $9.31^{\circ}$ & $9.96^{\circ}$ & $9.49^{\circ}$ \\
\hline Flame angle $\theta_{\tilde{c}=0.9}$ for $\tilde{c}=0.9$ at $\mathrm{C}$ & $10.54^{\circ}$ & $9.62^{\circ}$ & $10.22^{\circ}$ & $12.18^{\circ}$ \\
\hline
\end{tabular}

It can be seen from Fig. 5 that the contours of $\tilde{c}$ are mildly curved for the cases considered here. However, this trend weakens with increasing $\tilde{c}$ value, which can be substantiated from $\theta_{\tilde{c}=0.1}$, $\theta_{\tilde{c}=0.5}, \theta_{\tilde{c}=0.9}$ values listed in Table 5 . The flame angle $\theta_{\tilde{c}=0.9}$ for droplet cases is found to be smaller than that for the gaseous premixed case, whereas the flame angle $\theta_{\tilde{c}=0.1}$ for droplet cases remains larger than that for the gaseous premixed case at most locations. These differences originate as a result of the differences in flame brush thicknesses reported in Table 4, which in turn originate due to the differences between the composition of the gaseous mixture within the flame and the evolution characteristics of turbulence within the flame brush. It can be seen from Table 5 that $\theta_{\tilde{c}=0.1}$ changes appreciably with the axial distance and a similar qualitative trend is observed for $\theta_{\tilde{c}=0.5}$ but the variation of $\theta_{\tilde{c}=0.5}$ in the axial distance is much weaker than $\theta_{\tilde{c}=0.1}$. Moreover, $\theta_{\tilde{c}=0.9}$ does not change much in the axial direction for the cases considered here. In order to explain this behaviour, the PDFs of $\phi_{g}$ on $\tilde{c}=0.1,0.5$ and 0.9 isosurfaces at different axial locations are shown in Fig. 6. It is evident from Fig. 6 that the PDFs of $\phi_{g}$ are considerably different for different droplet diameters with higher probabilities of finding fuel-lean mixture for bigger droplet diameters. However, Fig. 6 shows that the mean value of $\phi_{g}$ increases in the downstream direction but the difference in the PDFs of $\phi_{g}$ 
decreases with increasing $\tilde{c}$, and with the axial distance because of the increased extents of droplet evaporation. Thus, the increase in the mean value of $\phi_{g}$ in the downstream direction is most prominent for $\tilde{c}=0.1$, which gives rise to its curved contours and relatively significant variations of $\theta_{\tilde{c}=0.1}$ in the axial direction. Figure 6 reveals that the mean $\phi_{g}$ values for $\tilde{c}=0.9$ contours do not change appreciably in the downstream direction and therefore the contours of $\tilde{c}=0.9$ remain straight for the cases considered here. Moreover, the gaseous equivalence ratio $\phi_{g}$ values remain comparable for $\tilde{c}=0.5$ and 0.9 for different droplet diameters and therefore these contours almost coincide with each other for different values of $a_{d} / \delta_{s t}$. It is worth noting that the variation of the PDFs shown in Fig. 6 in the flow direction remains weaker than that in Fig. 3. In Fig. 6, the PDFs of $\phi_{g}$ are shown for a given $\tilde{c}=\bar{\rho}^{-1} \int_{0}^{1.0} \rho c P(c) d c$ isosurfaces where $P(c)$ is the PDF of $c$ and $\bar{\rho}$ is the mean density. Thus $\phi_{g}$ from different $c$ values contribute to this and the gaseous phase equivalence ratio $\phi_{g}$ values for small values of $c$ are expected to be small. Consequently, the PDFs of $\phi_{g}$ from locations A to C in Fig. 6 do not change as much as the PDFs in Fig. 3 change from the location A to location C.

The flame angles and flame brush thicknesses are dependent on the reacting mixture composition in the gaseous phase, flame wrinkling and flame propagation rates. Thus, it is worthwhile to examine the statistical behaviours of gaseous equivalence ratio $\phi_{g}$, flame curvature $\kappa_{m}$, mode of combustion and local consumption and density-weighted displacement speeds (i.e. $S_{c}$ and $S_{d}^{*}$ ). 

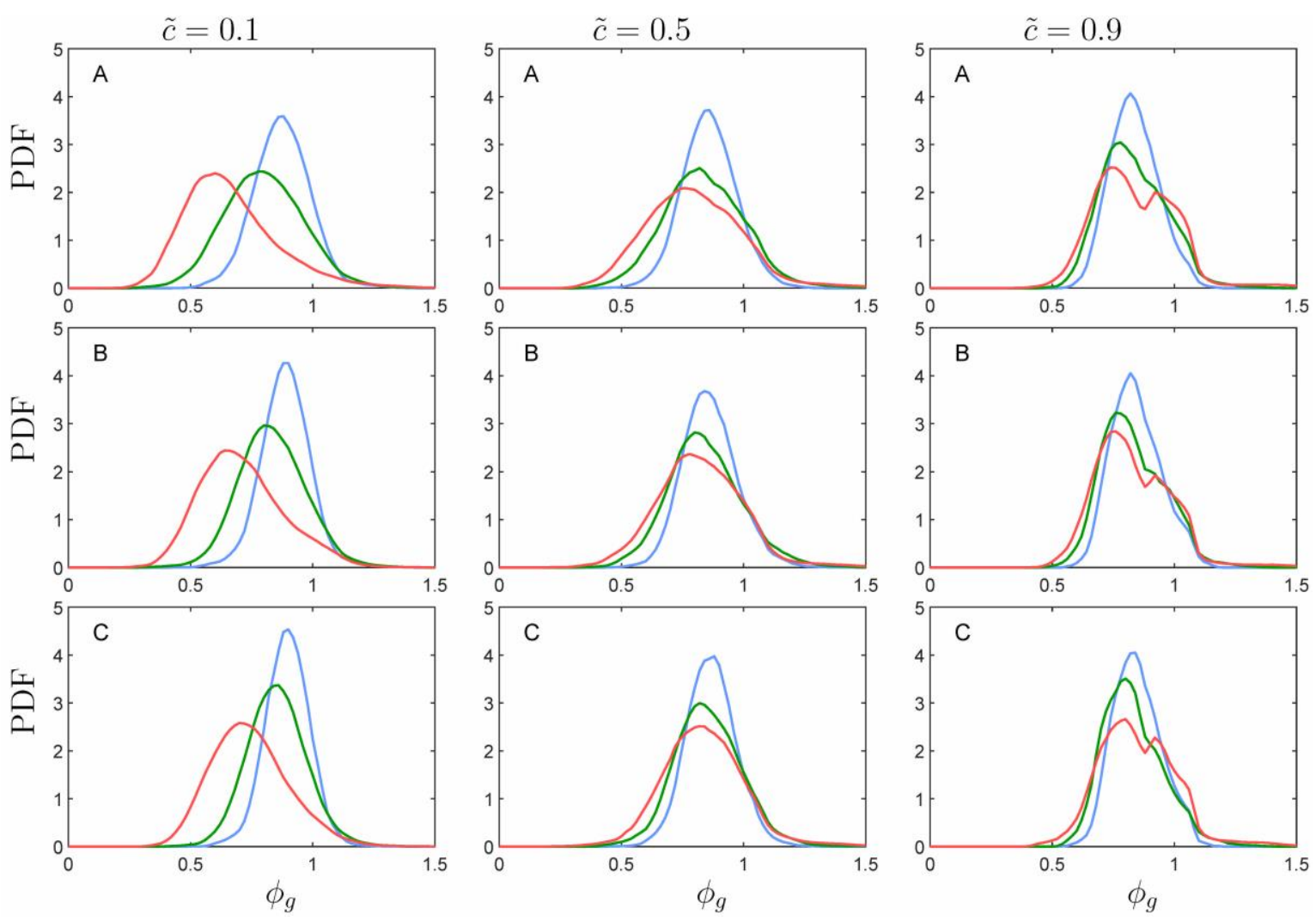

Figure 6. PDFs of $\phi_{g}$ conditional on $\tilde{c}=0.1,0.5,0.9$ isosurfaces at different locations A, B

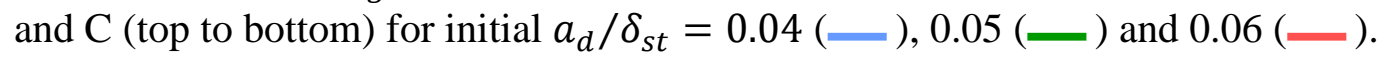

The presence of droplets significantly affects the flame morphology and induces flame wrinkling due to flame-droplet interaction (Lawes and Saat, 2011). In order to understand the flame-droplet interaction, the instantaneous views of $c=0.5$ isosurfaces, coloured by local values of flame curvature $\kappa_{m} \times \delta_{s t}$ for all cases considered here are shown in Fig. 7. The local curvature $\kappa_{m}$ is defined as $\kappa_{m}=\nabla \cdot \vec{N} / 2$ where $\vec{N}=-\nabla c /|\nabla c|$ is the local flame normal vector. According to these definitions, an element of the flame surface, which is convex (concave) to the reactants, has a positive (negative) curvature and the flame normal vector points towards the reactants. It can be seen from Fig. 7 that the flame surface remains smooth for the gaseous premixed flame case, and also for the droplet case with small droplets, which evaporate rapidly ahead of the flame and do not significantly interact with the flame surface. By contrast, the flame surfaces are significantly affected by large droplets and exhibit dimples 
in addition to the flame wrinkles induced by the turbulent flow field. The occurrences of flamedroplet interaction events increase in the downstream direction for larger droplets because they can survive longer in the domain due to their lower evaporation rate and bigger size.
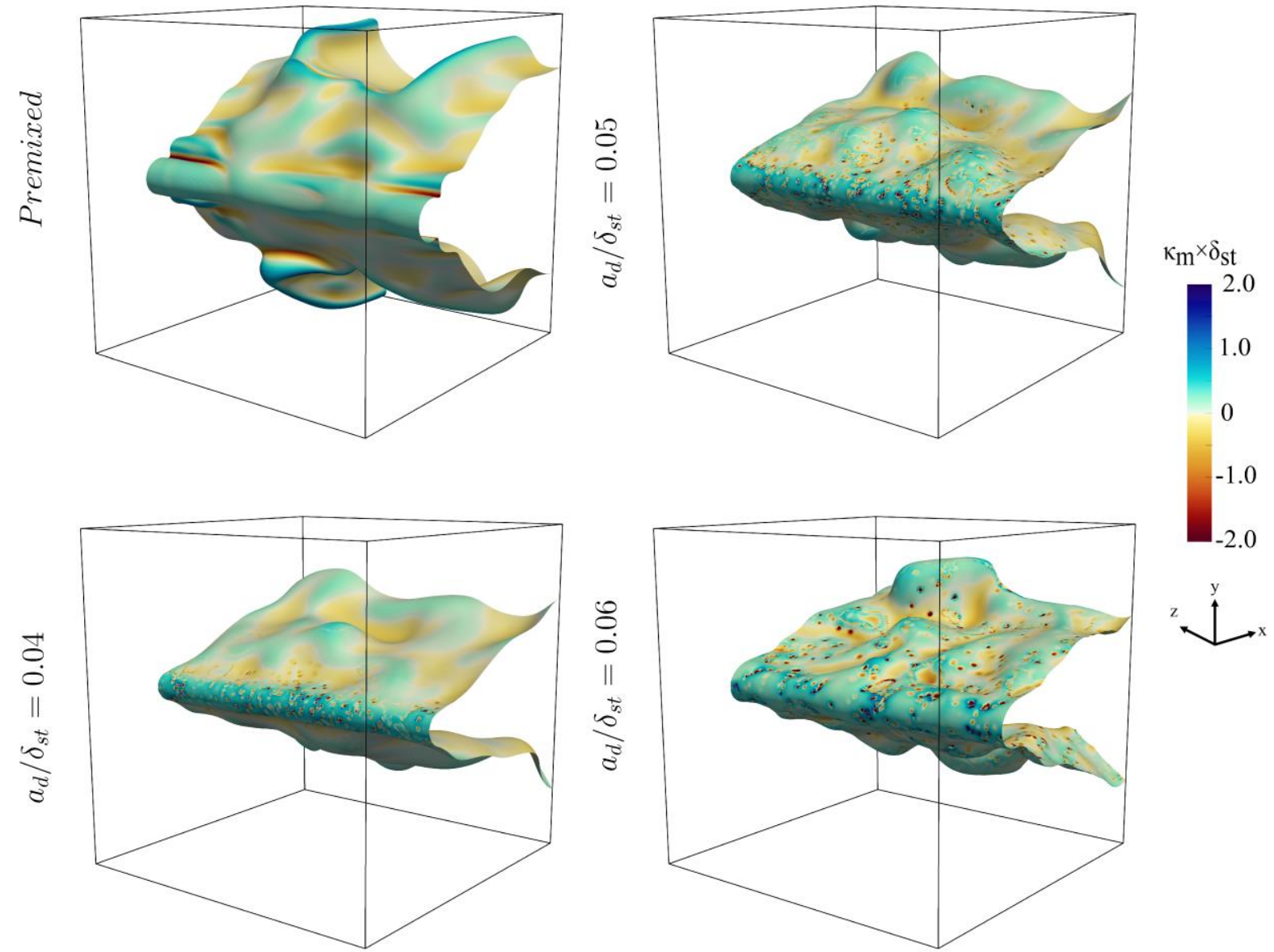

Figure 7. Instantaneous views of $c=0.5$ isosurface coloured with $\kappa_{m} \times \delta_{s t}$ values for gaseous premixed and spray flames with initial $a_{d} / \delta_{s t}=0.04,0.05,0.06$ at $t=2.0 t_{\text {flow }}$. 

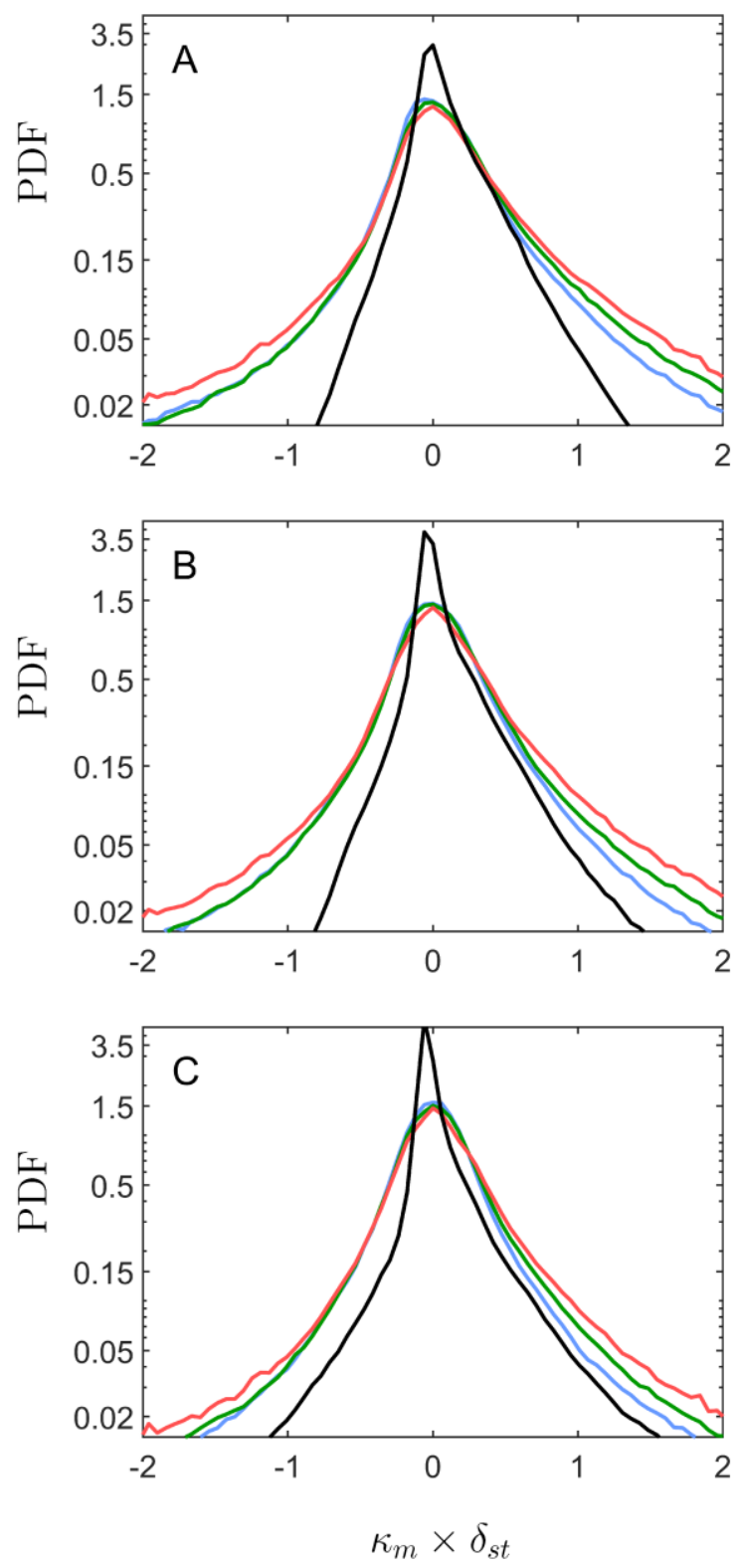

Figure 8. PDFs of $\kappa_{m} \times \delta_{s t}$ of the $c=0.8$ isosurface at locations A, B and C (top to bottom) for gaseous premixed ( $(-)$ ) and V-shaped spray flames with initial $a_{d} / \delta_{s t}=0.04(-)$, 0.05 ( -$)$ and $0.06(-)$.

The PDFs of local normalised flame curvature $\kappa_{m} \times \delta_{s t}$ of the $c=0.8$ isosurface at different planes (i.e. A, B and C) for all droplet cases and the gaseous premixed flame case considered in this study are shown in Fig. 8. The $c=0.8$ isosurface has been chosen because the reaction rate of progress variable attains its maximum value close to this value of $c$ for the present thermo-chemistry (Ozel-Erol et al., 2018) and thus this isosurface will henceforth be considered 
to be the flame surface in this analysis. However, the PDFs of local normalised flame curvature $\kappa_{m} \times \delta_{s t}$ for other reaction progress variable isosurfaces remain qualitatively similar to the $c=$ 0.8 isosurface. The PDFs of $\kappa_{m} \times \delta_{s t}$ peak at around zero value and show similar widths for all droplet cases at locations A, B and C. However, the width on the PDF of $\kappa_{m} \times \delta_{s t}$ marginally increases with increasing $a_{d} / \delta_{s t}$, which bears the signature of droplet-induced flame wrinkling for larger droplets. This behaviour is consistent with previous computational findings for spherically expanding spray flames (Ozel-Erol et al., 2018) for weak turbulence intensities. Furthermore, a comparison between gaseous premixed and droplet cases reveals that PDFs of $\kappa_{m} \times \delta_{s t}$ for spray flames have a wider distribution at planes A and B where droplet-flame interactions mainly occur. However, the difference in the distributions of $\kappa_{m} \times \delta_{s t}$ between spray and gaseous premixed flame cases decreases in the flow direction because droplets increasingly evaporate in the axial direction and flame-droplet interaction becomes less frequent. The differences in the widths of $\kappa_{m} \times \delta_{s t}$ PDFs are consistent with the differences in flame brush thicknesses for different droplets.

The mode of combustion in these flames can be characterised by the flame index $\mathrm{FI}=\left(\nabla Y_{F}\right.$. $\left.\nabla Y_{O}\right) /\left(\left|\nabla Y_{F}\right|\left|\nabla Y_{O}\right|\right)$ (Yamashita et al., 1996) so that a positive value of FI indicates a premixed mode of combustion, whereas a negative flame index FI indicates a non-premixed mode of combustion. The percentages of heat release rate arising from premixed and non-premixed modes of combustion are demonstrated in Fig. 9 for all droplet cases considered here at locations A, B and C. Figure 9 indicates that for all cases heat release mostly takes place under premixed mode at all locations. Thus, the gaseous phase combustion in these cases takes place mostly in stratified mixtures. However, it can be discerned from Fig. 9 that the percentage of overall heat release due to non-premixed combustion is greater in extent in the case with initial $a_{d} / \delta_{s t}=0.06$ than the corresponding cases with initial $a_{d} / \delta_{s t}=0.04$ and 0.05 . A careful 
examination of Fig. 3 reveals that there is a small peak at $\phi_{g}=1.0$ in the PDFs of $\phi_{g}$ for the initial $a_{d} / \delta_{s t}=0.06$ case, which originates due to non-premixed mode of combustion. Due to slow evaporation rate of large droplets the initial $a_{d} / \delta_{s t}=0.06$ case gives rise to higher likelihood of obtaining local islands of high and low fuel concentration than the other corresponding cases with smaller droplets, and this gives rise to the strengthening of nonpremixed mode of combustion in the case of large droplets (e.g. cases with initial $a_{d} / \delta_{s t}=0.06$ ). A small number of droplets in the case of large droplet diameter (e.g. cases with initial $\left.a_{d} / \delta_{s t}=0.06\right)$. escape through the flame due to turbulent motion and complete the evaporation in the high temperature zone in the region with $c=1.0$. Some of these unburned fuel-pockets eventually diffuse into the flame and mix with the air diffusing from the unburned gas side to give rise to local diffusion mode of burning.

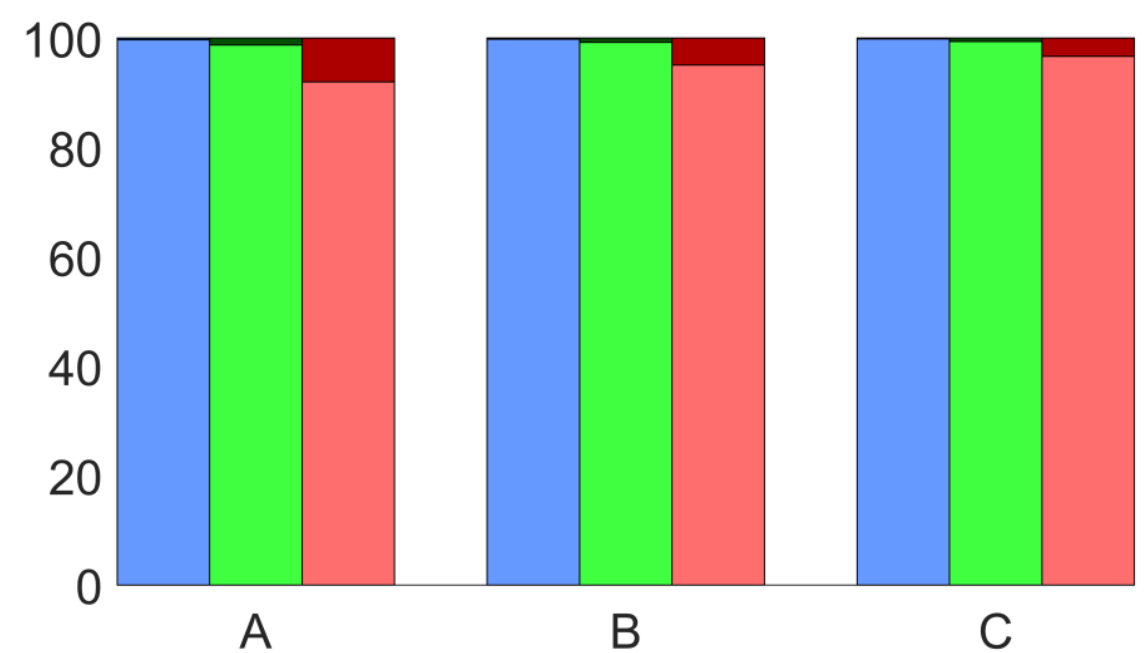

Figure 9. Percentage of heat release arising from premixed (for initial $a_{d} / \delta_{s t}=0.04(\square), 0.05$ $(\square), 0.06(\square)$ ) and non-premixed (for initial $a_{d} / \delta_{s t}=0.04(\square), 0.05(\square), 0.06(\square)$ ) modes of combustion at locations $\mathrm{A}, \mathrm{B}$ and $\mathrm{C}$ (left to right).

As the heat release takes place predominantly under the premixed mode, it is necessary to analyse the flame propagation statistics for the flames considered here. For this reason, it is worthwhile to analyse the terms on the right-hand side of the transport equation of the reaction 
progress variable $c$ (see Eq. 7), as they govern the statistical behaviours of density-weighted displacement speed $S_{d}^{*}$. The mean values of the terms $\dot{w}_{c}, \dot{S}_{l i q, c}, \dot{A}_{c}$ and $\nabla \cdot(\rho D \nabla c)$ conditional on $c$ for locations $\mathrm{A}, \mathrm{B}$ and $\mathrm{C}$ are shown in Fig. 10. The terms contributing the flame displacement speed $S_{d}$ are ensemble averaged for a given value of $c$ for 30 equally spaced bins of $c$ between 0.01 and 0.99 , and these values are subsequently time-averaged before presenting in Fig. 10. The same approach was adopted in several previous analyses (Boger et al., 1998; Chakraborty, 2007; Chakraborty and Cant, 2004,2005; Neophytou et al., 2010; 2012; Wacks et al., 2016).

Figure 10 suggests that the mean molecular diffusion term $\nabla \cdot(\rho D \nabla c)$ takes positive values in the preheat zone (i.e. $c<0.5$ ), whereas it assumes negative values and remains in approximate balance with the positive mean value of $\dot{w}_{c}$ in the reaction zone (i.e. $0.5 \leq c \leq 0.9$ ) for both gaseous premixed and spray V-shaped flames. It is evident from Fig. 10 that the mean values of $\dot{w}_{c}$ for droplets cases are smaller than the gaseous premixed case. This trend originates due to slower burning of stratified mixtures with $\phi_{g}<1.0$ and $\phi_{g}>1.0$ in the droplet cases than in the stoichiometric (i.e. $\left.\phi_{g}=1.0\right)$ premixed flame. The mean values of $\dot{w}_{c}$ decreases with increasing droplet diameter due to the increased propensity of fuel-lean burning in the gaseous phase. Furthermore, the mean values for all terms show similar trends at locations A, B, C for all droplet diameters. The mean values of the terms arising due to droplet evaporation $\dot{S}_{l i q, c}$ and mixture inhomogeneity $\dot{A}_{c}$ remain much smaller in magnitude in comparison to the magnitudes of the mean values of $\dot{w}_{c}$ and $\nabla \cdot(\rho D \nabla c)$ throughout the flame, which is consistent with previous findings for statistically planar (Wacks et al., 2016) and spherically expanding (OzelErol et al., 2019) flames. It can further be seen from Fig. 10 that the mean value of $\rho S_{d}|\nabla c|=$ $\left[\nabla \cdot(\rho D \nabla c)+\dot{w}_{c}+\dot{S}_{l i q, c}+\dot{A}_{c}\right]$ remains smaller than the mean value of $\rho_{0} S_{b, s t}|\nabla c|$ for all 
cases considered here in spite of having an overall equivalence ratio of unity (i.e. $\phi_{o v}=1.0$ ) in the unburned gas. This suggests that the surface-weighted value of density-weighted displacement speed (i.e. ratio of averaged/filtered values of $S_{d}^{*}|\nabla c|$ and $|\nabla c|$ ) (Trouvé and Poinsot, 1994; Boger et al., 1998) in all cases remains smaller than $S_{b, s t}$ due to the predominance of the fuel-lean mixture within the flame in spite of having $\phi_{o v}=1.0$ in the unburned gas. Figure 10 also reveals that the mean value of $\rho_{0} S_{b, s t}|\nabla c|$ decreases with increasing droplet diameter. The inverse of the maximum mean value of $|\nabla c|$ provides a measure of the flamelet thickness (i.e. $\delta \sim 1 / \max |\nabla c|$ ) at a given location. The smaller peak mean value of $\rho_{0} S_{b, s t}|\nabla c|$ for larger droplets indicates a thicker flame because $\rho_{0} S_{b, s t}$ remains the same for all cases. As the probability of finding fuel-lean mixture increases for larger droplets, the small values of $S_{b\left(\phi_{g}\right)}$ are more likely for larger droplets and accordingly flamelet thickness (which scales as $\delta \sim \alpha_{T 0} / S_{b\left(\phi_{g}\right)}$ ) increases with increasing droplet diameter. Thus, the occurrences of local flame thickening for large values of $a_{d} / \delta_{s t}$ in Fig. 2 is consistent with the observations regarding local flame thickening for large droplets. 

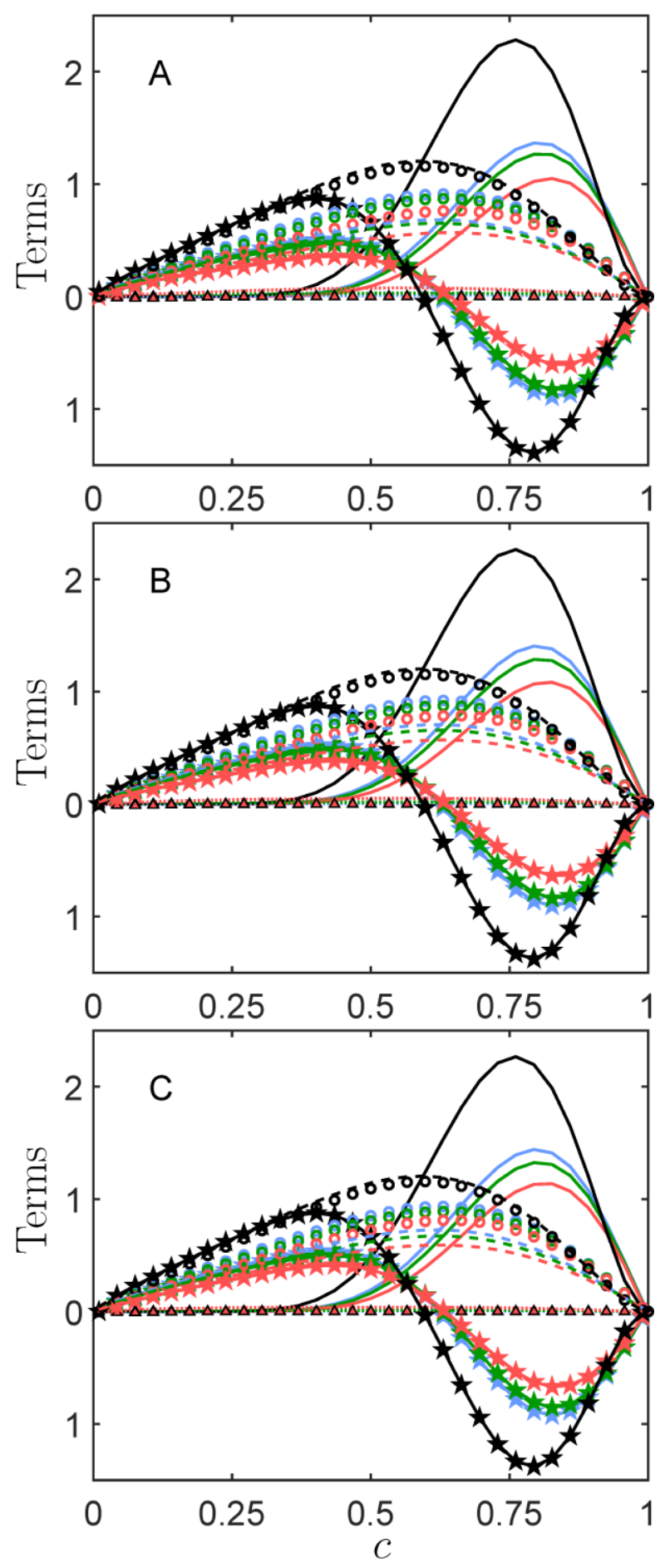

Figure 10. Variations of mean values of $\dot{w}_{c}$ (solid line), $\nabla \cdot(\rho D \nabla c)$ (line with star marker), $\dot{A}_{c}$ (dotted line), $\dot{S}_{l i q, c}$ (triangle marker), $\rho S_{d}|\nabla c|$ (dashed line) and $\rho_{0} S_{b, s t}|\nabla c|$ (circle) conditional on $c$ for premixed gaseous (-) and droplet cases with initial $a_{d} / \delta_{s t}=0.04$ (-), 0.05 ( $)$ and 0.06 (-) at locations A, B, C (top to bottom). All terms are normalised by $\delta_{s t} / \rho_{0} S_{b, s t}$.

The aforementioned behaviours of $\nabla \cdot(\rho D \nabla c), \dot{w}_{c}, \dot{S}_{l i q, c}, \dot{A}_{c}$ affect the statistical behaviours of 
$S_{d}^{*}$, whereas $\dot{w}_{c}$ and the flame thickness determine the behaviour of consumption speed $S_{c}$. The normalised mean values of consumption speed $S_{c} / S_{b, s t}$ and $S_{c} / S_{b\left(\left\langle\phi_{g}\right\rangle\right)}$ and density-weighted displacement speed, $S_{d}^{*} / S_{b, s t}$ and $S_{d}^{*} / S_{b\left(\left\langle\phi_{g}\right\rangle\right)}$ evaluated on the $c=0.8$ isosurface at locations $\mathrm{A}, \mathrm{B}$ and $\mathrm{C}$ are shown in Fig. 11 where $\left\langle\phi_{g}\right\rangle$ is the averaged equaivalence ratio on the $c=0.8$ isosurface at this location and $S_{b\left(\left\langle\phi_{g}\right\rangle\right)}$ is the laminar burning velocity corresponding to $\left\langle\phi_{g}\right\rangle$. As the consumption speed $S_{c}$ is directly dependent on $\dot{w}_{c}$, the mean values of $S_{c} / S_{b, s t}$ show a qualitatively similar trend as the mean values of $\dot{w}_{c}$. Therefore, the mean values of consumption speed for the gaseous premixed V-shaped flame case assume considerably greater values than those for droplet cases. However, the mean values of $S_{d}^{*} / S_{b, s t}$ remain different from the mean values of $S_{c} / S_{b, s t}$ due to the combined influences of $\dot{S}_{l i q, c}, \dot{A}_{c}, \nabla \cdot(\rho D \nabla c)$ and $\dot{w}_{c}$ (but predominantly due to $\nabla \cdot(\rho D \nabla c)$ and $\left.\dot{w}_{c}\right)$. The mean values of $S_{d}^{*} / S_{b, s t}$ for a given value of $a_{d} / \delta_{s t}$ remain comparable at locations $\mathrm{A}, \mathrm{B}$ and $\mathrm{C}$ but slight variations can be discerned between the mean values corresponding to these locations. The mean values of $S_{d}^{*} / S_{b, s t}$ remain close to unity for the gaseous premixed case. Moreover, the mean values of $S_{c} / S_{b, s t}$ show decreasing trends with increasing $a_{d} / \delta_{s t}$ because of the greater likelihood of obtaining fuellean mixtures for larger droplets (see Figs. 3 and 6).

Another alternative way to present the mean values of consumption and density-weighted displacement speeds is to normalise them with respect to the laminar burning velocity $S_{b\left(\left\langle\phi_{g}\right\rangle\right)}$ for the mean equivalence ratio $\left\langle\phi_{g}\right\rangle$ within the flame. However, the value of $S_{b\left(\left\langle\phi_{g}\right\rangle\right)}$ changes from one location to another and therefore, the relative magnitudes of $S_{c}$ and $S_{d}^{*}$ cannot be assessed from the values of $S_{c} / S_{b\left(\left\langle\phi_{g}\right\rangle\right)}$ and $S_{d}^{*} / S_{b\left(\left\langle\phi_{g}\right\rangle\right)}$. The values of $S_{b\left(\left\langle\phi_{g}\right\rangle\right)}$ remain smaller than $S_{b, s t}$ for all the droplet cases considered here and this tendency is most prominent for large droplet diameters (e.g. initial droplet size of $a_{d} / \delta_{s t}=0.06$ ). This can be seen from the wider 
PDFs of $\phi_{g}$ with increasing $a_{d} / \delta_{s t}$ in Figs. 3 and 6, and therefore the probability of obtaining burning more reactive $\left.\phi_{g}(\approx 1.0)\right\rangle\left\langle\phi_{g}\right\rangle$ mixture increases with increasing droplet size in the droplet cases. This leads to increasing trends of $S_{c} / S_{b\left(\left\langle\phi_{g}\right\rangle\right)}$ and $S_{d}^{*} / S_{b\left(\left\langle\phi_{g}\right\rangle\right)}$ with increasing $a_{d} / \delta_{s t}$ in contrast to the variations of $S_{c} / S_{b, s t}$ and $S_{d}^{*} / S_{b, s t}$, and this behaviour is more prominent at location $\mathrm{A}$ than in locations $\mathrm{B}$ and $\mathrm{C}$ since the leaner combustion yields small laminar burning velocities at location A.

Table 6. Time averaged normalised values of $A_{s}=\int_{V}|\nabla c| d V, A_{p, \tilde{c}}, \Omega_{f}=\int_{V}\left|\dot{w}_{F}\right| d V /$ $\rho_{0} \delta_{s t}^{2} S_{b, s t}, S=\int_{V} \dot{w}_{c} d V /\left[\rho_{0} S_{b, s t} \int_{V}|\nabla c| d V\right]$ and $S^{\prime}=\int_{V} \dot{w}_{c} d V /\left[\rho_{0} S_{b\left(\phi_{f}\right)} \int_{V}|\nabla c| d V\right]$

\begin{tabular}{|c|c|c|c|c|}
\hline $\boldsymbol{a}_{\boldsymbol{d}} / \boldsymbol{\delta}_{\boldsymbol{s t}}$ & $\begin{array}{c}\text { Gaseous } \\
\text { Premixed } \\
\text { Flame }\end{array}$ & $\mathbf{0 . 0 4}$ & $\mathbf{0 . 0 5}$ & $\mathbf{0 . 0 6}$ \\
\hline$A_{s} / \delta_{s t}^{2}$ & 1797 & 1722 & 1684 & 1708 \\
\hline$A_{p, \tilde{c}=0.1} / \delta_{s t}^{2}$ & 1600 & 1494 & 1499 & 1498 \\
\hline$A_{p, \tilde{c}=0.5} / \delta_{s t}^{2}$ & 1495 & 1404 & 1408 & 1413 \\
\hline$A_{p, \tilde{c}=0.9} / \delta_{s t}^{2}$ & 1432 & 1350 & 1354 & 1358 \\
\hline$\Omega_{f}$ & 118 & 75 & 68 & 58 \\
\hline$S$ & 1.06 & 0.77 & 0.71 & 0.60 \\
\hline$S^{\prime}$ & 1.00 & 1.00 & 1.11 & 1.41 \\
\hline
\end{tabular}

The time-averaged values of flame surface area $A_{s}$, the normalised volume integrated fuel reaction rate magnitude $\Omega_{f}$ (the volume integrated reaction progress variable reaction rate $\Omega_{c}=$ $\int_{V} \dot{w}_{c} d V / \rho_{0} \delta_{s t}^{2} S_{b, s t}$ shows the same qualitative trend and thus is not shown here) and the normalised ratio of volume-integrated product formation rate to the flame surface area $S=$ $\int_{V} \dot{w}_{c} d V /\left[\rho_{0} S_{b, s t} \int_{V}|\nabla c| d V\right]$ are listed in Table 6, which shows that $A_{s}$ remains comparable for all cases with different initial droplet diameter. The probability of finding smaller values of $|\nabla c|$ is greater in the case with initial $a_{d} / \delta_{s t}=0.05$ than in the case with initial $a_{d} / \delta_{s t}=0.04$, which contributes to the smaller flame surface area $A_{s}$ for the initial $a_{d} / \delta_{s t}=0.05$ case than 
that in the $a_{d} / \delta_{s t}=0.04$ case. Although the probability of finding smaller values of $|\nabla c|$ is greater in the case with initial $a_{d} / \delta_{s t}=0.06$ than in the case with initial $a_{d} / \delta_{s t}=0.05$ but the larger droplets in the initial $a_{d} / \delta_{s t}=0.06$ case lead to more flame-wrinkling and increase the total flame volume to yield a flame area which is greater than that in the initial $a_{d} / \delta_{s t}=$ 0.05 case but still smaller than in the initial $a_{d} / \delta_{s t}=0.04$ case considered here. Furthermore, the time-averaged flame surface area of gaseous premixed V-shaped flame remains slightly larger than those for droplet cases.

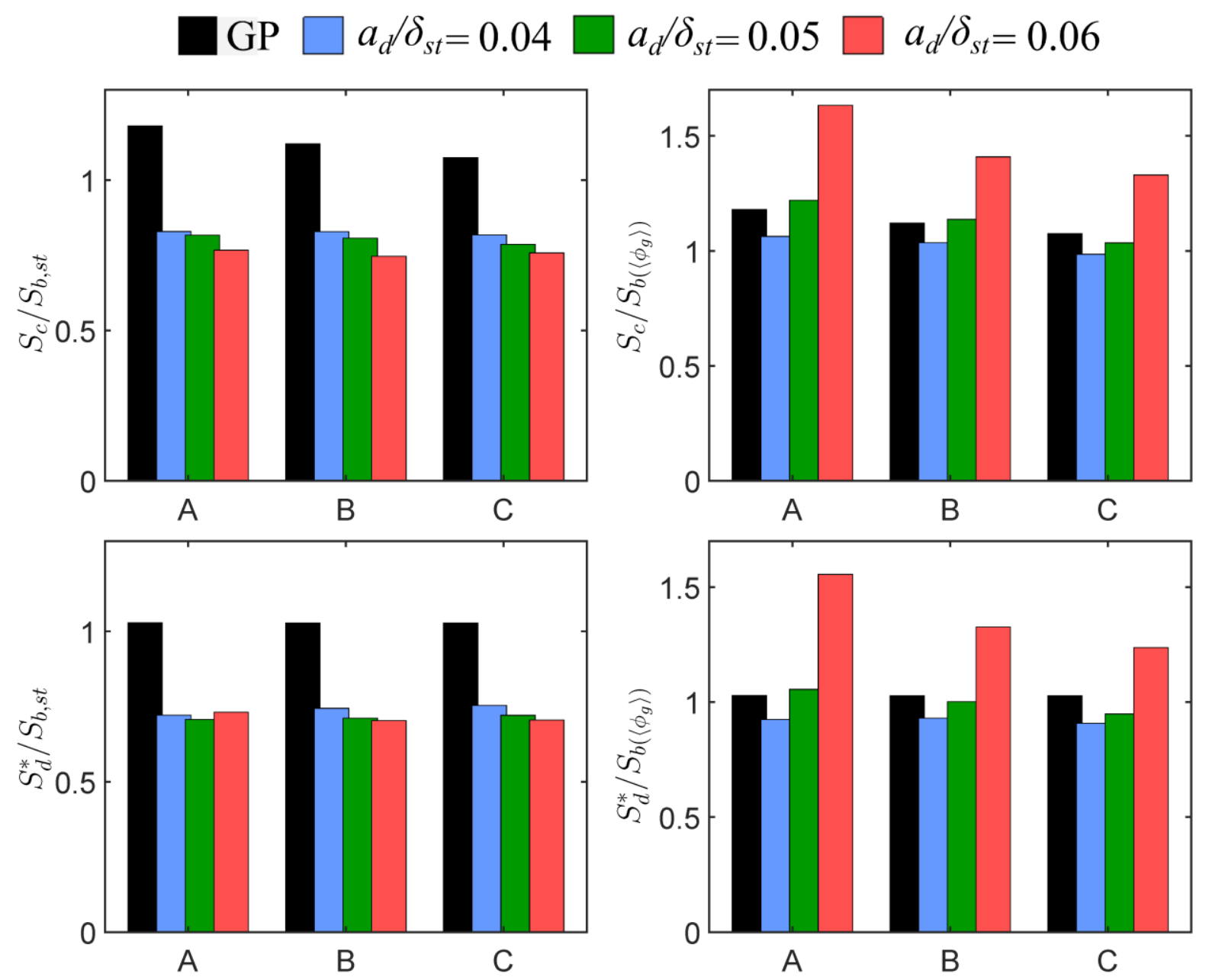

Figure 11. The mean values of $S_{c} / S_{b, s t}, S_{d}^{*} / S_{b, s t}, S_{c} / S_{b\left(\left\langle\phi_{g}\right\rangle\right)}$ and $S_{d}^{*} / S_{b\left(\left\langle\phi_{g}\right\rangle\right)}$ on the $c=0.8$ isosurface at locations A-C.

The time-averaged value of $\Omega_{f}$ decreases with increasing $a_{d} / \delta_{s t}$ as the probability of fuel-lean 
combustion increases with increasing droplet size, which is consistent with the decreasing trend of the mean values of $\dot{w}_{c}$ with increasing $a_{d}$ (see Fig. 10). The integral $\int_{V} \dot{w}_{c} d V$ can be expressed as: $\int_{V} \dot{w}_{c} d V \approx \rho_{0} S_{c} A_{s}$ and therefore $S_{c}<S_{b, s t}$ from Fig. 11 implies that $S=$ $\int_{V} \dot{w}_{c} d V /\left[\rho_{0} S_{b, s t} \int_{V}|\nabla c| d V\right]$ is expected to be smaller than unity for the cases considered here. The reduction of $\int_{V} \dot{w}_{c} d V$ dominates over the increase in flame surface area $A_{s}$ for large droplets and ultimately gives rise to a decreasing trend of $S$ with increasing $a_{d}$, which is also consistent with the decreasing trends of $S_{c} / S_{b, s t}$ with increasing $a_{d} / \delta_{s t}$. It can be seen from Table 6 that the ratio $S$ stays close to unity for the gaseous premixed $\mathrm{V}$-shaped flame, since the mean values of $\dot{w}_{c}+\nabla \cdot(\rho D \nabla c)$ and $\rho_{0} S_{b, s t}|\nabla c|$ remain close to each other (see Fig. 10).

It has been demonstrated in Fig. 9 that most of the heat release in these flames takes place due to the premixed mode of combustion. These flames were previously referred to as premixed sprays by Mizutani and Nishimoto (1972) and Silverman et al. (1993). It has been demonstrated in several previous analyses that the equality between the enhancements of burning rate and flame surface area in turbulent premixed flames with respect to their laminar counterparts (which is commonly referred to as Damköhler's first hypothesis) remains valid for statistically planar turbulent premixed flames even for the thickened flame regimes (i.e. for small (large) values of Damköhler (Karlovitz) number) (Aspden et al., 2011; Nivarti and Cant, 2017; Ahmed et al., 2019) even though Damköhler's first hypothesis (Damköhler, 1941) was originally proposed for large values of Damköhler number (i.e. $D a \gg 1$ ). As the combustion in these spray flames takes place in the thickened flame regime, it is worthwhile to assess if Damköhler's first hypothesis remains valid for these flames.

Figure 10 implies that the mean value of $\left[\dot{S}_{l i q, c}+\dot{A}_{c}\right]$ remains much smaller than the mean 
value of $\rho_{0} S_{d}^{*}|\nabla c|$. As $\int_{V} \nabla \cdot(\rho D \nabla c) d V=0$, the integral $\int_{V} \dot{w}_{c} d V$ can be approximated as:

$$
\int_{V} \dot{w}_{c} d V \approx \sum\left\langle\rho S_{d}|\nabla c| \mid c=c^{*}\right\rangle V_{c=c^{*}}
$$

where $\left\langle Q \mid c=c^{*}\right\rangle$ is the mean value of a general quantity $Q$ conditional upon $c=c^{*}$ and $V_{c=c^{*}}$ is the volume of the samples corresponding to $c=c^{*}$. It can be seen from Fig. 10 that the mean value of $\rho S_{d}|\nabla c|$ remains smaller than $\rho_{0} S_{b, s t}|\nabla c|$ which leads to $S<1.0$ for all cases. According to Damköhler's first hypothesis (Damköhler, 1940), the enhancements of burning rate and flame surface under turbulence are proportional to each other, which implies a unity value of $S$ (i.e. $S=1.0$ ) (Chakraborty et al., 2019). Thus, the non-unity values of $S$ indicate a departure from Damköhler's first hypothesis and suggest that Damköhler's first hypothesis in its original form is not valid for turbulent spray flames. It can further be seen from Table 6 that $S$ remains smaller than unity for all the droplet cases but $S^{\prime}=\int_{V} \dot{W}_{c} d V /\left[\rho_{0} S_{b\left(\phi_{f}\right)} \int_{V}|\nabla c| d V\right]$ (where $\phi_{f}$ is the volume-averaged gaseous phase equivalence ratio in the region corresponding to $0.01 \leq c \leq 0.99$ ) values are closer to unity and greater than the value of $S=$ $\int_{V} \dot{w}_{c} d V /\left[\rho_{0} S_{b, s t} \int_{V}|\nabla c| d V\right]$ because $S_{b\left(\phi_{-} f\right)} / S_{b, s t}$ remains smaller than unity. However, Table 6 suggests that the departure of $S^{\prime}$ from 1.0 can also be significant for the large droplets (e.g. initial droplet size of $a_{d} / \delta_{s t}=0.06$ ), and thus the conclusions regarding Damköhler's first hypothesis in its original form do not change if $S^{\prime}$ is used.

It is possible to define a turbulent burning velocity $S_{T}$ as: $S_{T}=\int_{V} \dot{w}_{c} d V /\left(\rho_{0} A_{p, \tilde{c}}\right)=$ $S\left(A_{s} / A_{p, \tilde{c}}\right) S_{b, s t}$ where $A_{p, \tilde{c}}$ is the flame area of the $\tilde{c}$ isosurface with respect to which $S_{T}$ is evaluated. The values of $A_{p, \tilde{c}=0.1}, A_{p, \tilde{c}=0.5}$ and $A_{p, \tilde{c}=0.9}$ for the cases considered here are listed in Table 6. It is evident from Table 6 that $A_{s}$ and $A_{p, \tilde{c}}$ assume comparable values for different values of $a_{d}$ and thus a decreasing trend of $S$ with increasing droplet diameter gives rise to a 
reduction in turbulent burning velocity $S_{T}$ with an increase in droplet diameter. This can be substantiated from Table 7 where $S_{T} / S_{b, s t}$ for the $\tilde{c}=0.1$ and 0.5 isosurfaces are presented. It is also worth noting that $S_{T} / S_{b, s t}$ remains smaller than unity (i.e. $S_{T} / S_{b, s t}<1.0$ ) for the droplet cases considered here but it should be considered that the normalised laminar burning velocity $S_{b\left(\left\langle\phi_{g}\right\rangle\right)} / S_{b, s t}$ for the mean gaseous phase equivalence ratio ranges between $0.78-0.83,0.67$ 0.76, 0.47-0.57 between locations $\mathrm{A}$ to $\mathrm{C}$ (with higher value at location $\mathrm{C}$ ) for initial values of $a_{d} / \delta_{s t}=0.04,0.05$ and 0.06 , respectively. This suggests that $S_{T} / S_{b\left(\left\langle\phi_{g}\right\rangle\right)}$ remains greater than unity for all cases as given in Table 7 and thus the overall burning rate gets enhanced under turbulence and this augmentation is the most prominent for the case with the largest droplets as this case experiences the largest value of $S_{T} / S_{b\left(\left\langle\phi_{g}\right\rangle\right)}$ among the flames considered here. It can be seen from Table 7 that turbulent burning velocity $S_{T}$ for the gaseous stoichiometric turbulent premixed flame assumes values greater than the corresponding laminar burning velocity (i.e. $\left.S_{T} / S_{b, s t}>1.0\right)$. This further suggests that the values of $S_{T} / S_{b, s t}$ for spray V-flames are smaller than the corresponding turbulent premixed gaseous flame and $S_{T} / S_{b, s t}$ decreases with increasing droplet diameter.

Table 7. Turbulent burning velocity $S_{T}$ based on the surface area for $\tilde{c}=0.1$ and 0.5 isosurfaces

\begin{tabular}{|c|c|c|c|c|}
\hline $\boldsymbol{a}_{\boldsymbol{d}} / \boldsymbol{\delta}_{\boldsymbol{s t}}$ & $\begin{array}{c}\text { Gaseous } \\
\text { Premixed } \\
\text { Flame }\end{array}$ & $\mathbf{0 . 0 4}$ & $\mathbf{0 . 0 5}$ & $\mathbf{0 . 0 6}$ \\
\hline$S_{T, \tilde{c}=0.1} / S_{b, s t}$ & 1.19 & 0.88 & 0.80 & 0.69 \\
\hline$S_{T, \tilde{c}=0.1} / S_{b,\left(\left\langle\phi_{g}\right\rangle, A\right)}$ & - & 1.13 & 1.19 & 1.47 \\
\hline$S_{T, \tilde{c}=0.1} / S_{b,\left(\left\langle\phi_{g}\right\rangle, B\right)}$ & - & 1.10 & 1.13 & 1.30 \\
\hline$S_{T, \tilde{c}=0.1} / S_{b,\left(\left\langle\phi_{g}\right\rangle, C\right)}$ & - & 1.06 & 1.05 & 1.21 \\
\hline$S_{T, \tilde{c}=0.5} / S_{b, s t}$ & 1.27 & 0.94 & 0.85 & 0.73 \\
\hline$S_{T, \tilde{c}=0.5} / S_{b,\left(\left\langle\phi_{g}\right\rangle, A\right)}$ & - & 1.21 & 1.27 & 1.55 \\
\hline$S_{T, \tilde{c}=0.5} / S_{b,\left(\left\langle\phi_{g}\right\rangle, B\right)}$ & - & 1.18 & 1.20 & 1.38 \\
\hline$S_{T, \tilde{c}=0.5} / S_{b,\left(\left\langle\phi_{g}\right\rangle, C\right)}$ & - & 1.13 & 1.12 & 1.28 \\
\hline
\end{tabular}




\section{CONCLUSIONS}

The mode of combustion, droplet-induced flame wrinkling, mean behaviours of different terms of the reaction progress variable transport equation, and flame propagation statistics for $\mathrm{V}$ shaped flames in a droplet-laden mixture of an overall (i.e. liquid+gaseous) equivalence ratio of unity have been investigated for different droplet diameters using three-dimensional DNS data. The flame behaviour has been compared with a turbulent gaseous stoichiometric premixed V-shaped flame under same flow conditions. It has been found that the flame brush thickness is affected by both droplet diameter and the axial distance from the flame holder and it increases with decreasing droplet diameter and with increasing axial distance. Furthermore, the flame brush has been found to be thicker in droplet cases than in the corresponding gaseous premixed flame case. The predominance of finding fuel-lean mixture within the flame decreases in the downstream direction due to evaporation of droplets and this effect is particularly strong for large droplets. The interaction of droplets with the flame surface gives rise to dimples on the reaction progress variable isosurfaces. However, droplet-induced flame wrinkling contributes to the widening of flame curvature PDFs, and also acts to increase the total flame surface area for large droplets. The mean values of the reaction rate of progress variable decrease with increasing droplet diameter. Furthermore, the mean value of the reaction rate of progress variable for a given droplet diameter shows similar magnitudes at different axial locations for all droplet cases. A similar qualitative trend has been observed for the axial distance dependences of the mean values of consumption speed and density-weighted displacement speed for a given value of droplet diameter. The mean values of consumption speed are found to decrease with increasing droplet diameter because of the greater likelihood of fuel-lean combustion in large droplet cases. By contrast, the mean density-weighted displacement speed remains comparable for all the droplet sizes considered here. Recent DNS analyses in statistically planar flames (Wacks et al., 2016) and spherically expanding flames (Ozel-Erol et 
al., 2018,2019) under both laminar and weakly turbulent conditions revealed significant differences in flame curvature, density-weighted displacement speed and consumption speed in response to droplet diameter, whereas the effects of droplet diameter are relatively weaker in V-flames than in statistically planar and spherically expanding flames. For the cases with globally stoichiometric equivalence ratio (Ozel-Erol et al., 2019), the mean values of densityweighted displacement speed were found to exhibit a mild increase with the increasing droplet diameter whereas the mean values of consumption speed showed a decreasing trend with the increasing droplet diameter. The statistically planar and spherically expanding flames interact with droplets at all stages of their propagation into the unburned gas (Wacks et al., 2016; OzelErol et al., 2018,2019) but in turbulent V-spray flames, the droplets evaporate in the mean flow direction. Therefore, the frequency of flame-droplet interaction decreases and the mean value of $\phi_{g}$ increases in the downstream direction.

It has been demonstrated that in turbulent droplet-laden V-shaped flames, the enhancement of burning rate under turbulence cannot be equated to the enhancement of the flame surface area with respect to the corresponding quantities under laminar conditions. Moreover, the turbulent burning velocity has been found to decrease with increasing droplet diameter due to the reduction in the volume-integrated reaction rate of reaction progress variable as a result of the greater likelihood of finding fuel-lean gaseous mixture for larger droplets. Although several previous analyses revealed that the flame speed statistics for turbulent premixed flames obtained from simple chemistry (Chakraborty and Cant, 2004, 2005; Chakraborty, 2007) remain in good agreement with the corresponding results obtained from a detailed chemical mechanism (Peters et al., 1998; Echekki and Chen, 1999), further analyses in the presence of a detailed chemical mechanism will be necessary for confirming the current findings and obtaining more comprehensive physical understanding regarding the roles played by the 
intermediate species. The distance of the flame holder from the inlet can also potentially have an influence on the results, but the gaseous phase equivalence ratio remains small for the $x$ locations before the flame holder and thus it can reasonably be expected that the qualitative nature of the results are unlikely to change with the variation of the distance of the flame holder from the inlet. However, further study in this respect will be necessary. The aforementioned challenges will form the basis of future investigations.

\section{ACKNOWLEDGEMENTS}

The financial support of the Republic of Turkey Ministry of National Education and EPSRC (EP/K025163/1, EP/R029369/1) and the computational support of Rocket and ARCHER are gratefully acknowledged. 


\section{REFERENCES}

Ahmed, U., Chakraborty, N., Klein, M., 2019. Insights into the bending effect in premixed turbulent combustion using the Flame Surface Density transport, Combust. Sci. Technol., 191, 898-920.

Alshaalan, T.M., Rutland, C. J., 1998. Turbulence, scalar transport, and reaction rates in flamewall interaction. Proc. Coombsut. Inst. 28, 793-799.

Aspden, A. J., Day, M. S., Bell, J. B., 2011. Turbulence-flame interactions in lean premixed hydrogen: transition to the distributed burning regime, J. Fluid Mech., 680,287-320.

Ballal, D.R., Lefebvre, A.H., 1981. Flame propagation in heterogeneous mixtures of fuel droplets, fuel vapor and air. Proc. Combust.Inst. 18, 321-328.

Bell, J.B., Day, M.S., Shepherd, I.G., Johnson, M.R., Cheng, R.K., Grcar, J. F., Beckner, V.E., Lijewski, M., 2005. Numerical simulation of a laboratory-scale turbulent V-flame. P. Natl. A Boger, M., Veynante, D., Boughanem, H., and Trouvé, A., 1998. Direct Numerical Simulation analysis of flame surface density concept for Large Eddy Simulation of turbulent premixed combustion, Proc. Combust. Inst., 27, 917-925.

Burgoyne, J.H., Cohen, L., 1954. The effect of drop size on flame propagation in liquid aerosols. Proc. R. Soc. London. Ser. A. 225, 375-392.

Bray, K.N.C., 1980. Turbulent flows with premixed reactants, in Turbulent Reacting Flows. Springer Verlag, Berlin Heidelburg, New York, eds. Libby, P.A., and Williams, F.A., pp. 115183.

Chaos, M., Kazakov, A., Zhao, Z., Dryer, F.L., 2007. A high - temperature chemical kinetic model for primary reference fuels. Int. J. Chem. Kinet. 39, 399- 414.

Chakraborty, N., Cant, R.S., 2004. Unsteady effects of strain rate and curvature on turbulent premixed flames in an inlet-outlet configuration. Combust. Flame 137, 129-147.

Chakraborty, N., Cant, R.S., 2005. Influence of Lewis Number on curvature effects in turbulent 
premixed flame propagation in the thin reaction zones regime. Phys. Fluids 17,105105.

Chakraborty, N., 2007. Comparison of displacement speed statistics of turbulent premixed flames in the regimes representing combustion in corrugated flamelets and thin reaction zones. Phys. Fluids 19,105109.

Chakraborty, N., Hawkes, E.R., 2008. Chen, J.H., Cant, R.S., Effects of strain rate and curvature on Surface Density Function transport in turbulent premixed CH4-air and H2-air flames, A comparative study, Combust. Flame, 154,259-280.

Chakraborty, N., Alwazzan, D., Klein, M., Cant, R.S., 2019. On the validity of Damköhler's first hypothesis in turbulent Bunsen burner flames: A computational analysis. Proc. Combust. Inst. 37, 2231-2239.

Chen, J.H., Im, H.G., 1998. Correlation of flame speed with stretch in turbulent premixed methane/air flame, Proc. Combust. Inst., 27, 819-826.

Chiu, H.H., Liu, T.M., 1977. Group combustion of liquid droplets. Combust. Sci. Technol. 17(3-4), 127-142.

Damköhler, G., 1940. Zeitschrift fur Elektrochemie und angewante physikalische. Chemie 46, 601-626.

de Chaisemartin, S., Fréret, L., Kah, D., Laurent, F., Fox, R., Reveillon, J., 2009. Eulerian models for turbulent spray combustion with polydispersity and droplet crossing. $\mathrm{C} \mathrm{R}$ Mecanique 48, 337-438

Domingo, P., Vervisch, L., Payet, S., Hauguel, R., 2005. DNS of a premixed turbulent V flame and LES of a ducted flame using a FSD-PDF subgrid scale closure with FPI-tabulated chemistry. Combust. Flame 143, 566-586.

Dunstan, T. D., Swaminathan, N., Bray, K. N. C., Cant, R. S., 2011. Geometrical Properties and Turbulent Flame Speed Measurements in Stationary Premixed V-flames Using Direct 
Numerical Simulation. Flow Turb. Combust. 87, 237-259.

Dunstan, T. D., Swaminathan, N., Bray, K. N. C., 2012. Influence of flame geometry on turbulent premixed flame propagation: a DNS investigation. J. Fluid Mech. 709, 191-222.

Echekki, T., Chen, J.H., 1996. Unsteady Strain rate and curvature effects in turbulent premixed methane-air flames. Combust. Flame. 106, 184-202.

Echekki, T., Chen, J.H., 1999. Analysis of the contribution of curvature to premixed flame propagation. Combust. Flame 118, 303-311.

Fujita, A., Watanabe, H., Kurose, R., Komori, S., 2013. Two-dimensional direct numerical simulation of spray flames - Part 1: Effects of equivalence ratio, fuel droplet size and radiation, and validity of flamelet model. Fuel 104, 515-525.

Gouldin, F.C., 1996. Combustion intensity and burning rate integral of premixed flames. Proc. Combust. Inst. 26, 381-388.

Greenberg, J.B., Silverman, I., Tambour, Y., 1998. On droplet enhancement of the burning velocity of laminar premixed spray flames. Combust. Flame 113, 271-273.

Han, I., Huh, K.Y., 2008. Roles of displacement speed on evolution of flame surface density for different turbulent intensities and Lewis numbers in turbulent premixed combustion. Combust. Flame, 152, 194-205.

Haruki, Y., Pillai, A.L., Kitano, T. and Kurose, R., 2018. Numerical investigation of flame propagation in fuel droplet arrays., At. Sprays 28, 357-388.

Hayashi, S., Kumarevgai, S., Sakai, T., 1977. Propagation velocity and structure of flames in droplet-vapor-air mixtures. Combust. Sci. Technol. 15, 169-177.

Haworth, D.C, Poinsot, T.J., 1992. Numerical simulations of Lewis number effects in turbulent premixed flames. J. Fluid Mech. 244, 405-436.

Hawkes, E.R.; Chen, J.H., 2004. Direct numerical simulation of hydrogen-enriched lean premixed methane air flames. Combust. Flame, 138, 242-258. 
Hawkes, E.R., Chen, J.H., 2005. Evaluation of models for flame stretch due to curvature in the thin reaction zones regime. Proc. Combust. Inst.,30, 647-655.

Hélie, J., Trouvé, A., 1998. Turbulent flame propagation in partially premixed combustion, Proc. Combust. Inst. 27, 891-898.

Jenkins, K. W., Klein, M., Chakraborty, N., Cant, R. S., 2006. Effects of strain rate and curvature on the propagation of a spherical flame kernel in the thin-reactionzones regime. Combust. Flame, 145, 415-434.

Kheirkhah, S, Gulder, O.L., 2014. Topology and brush thickness of turbulent premixed Vshaped flame. Flow, Turb. Combust. 93, 439-469.

Klein, M., Chakraborty, N., Jenkins, K.W., Cant, R.S., 2006. Effects of initial radius on the propagation of spherical premixed flame kernels in turbulent environment, Phys. Fluids, 18, 055102.

Kumar, K., Freeh, J. E., Sung, C. J. and Huang,Y., 2007. Laminar flame speeds of preheated iso-octance/O2/N2 and n-heptane/O2/N2 mixtures, J. Propulsion Power 23, 428-436.

Kuo, K.K., Acharya, R., 2012. Fundamentals of turbulent and multiphase combustion. John Wiley \& Sons, Inc., Hoboken, New Jersey, U.S.A.

Langevin, P., 1908. Sur la th'eorie du mouvement brownien. C. R. Acad. Sci. Paris 146, 530533.

Lawes, M., Saat, A., 2011. Burning rates of turbulent iso-octane aerosol mixtures in spherical flame explosions. Proc. Combust. Inst. 33, 2047-2054.

Letty, C., Renou, B., Reveillon, J., Saengkaew, S., Gréhan, G., 2013. Experimental study of droplet temperature in a two-phase heptane/air V-flame. Combust. Flame 160, 1803- 1881.

Malkeson, S.P., Chakraborty, N., 2010. Statistical analysis of displacement speed in turbulent stratified flames: A direct numerical simulation study. Combust. Sci. Technol. 182, 1841-1883. Meneveau, C., Poinsot, T., 1991. Stretching and quenching of flamelets in premixed turbulent 
combustion, Combust. Flame 86, 311-332.

Mizutani, Y., Nishimoto, T., 1972. Turbulent flame velocities in premixed sprays part i. experimental study. Combust. Sci. Technol. 6, 1-10.

Nakamura, M., Akamatsu, F., Kurose, R., Katsuki, M., 2005. Combustion mechanism of liquid fuel spray in a gaseous flame. Phys. Fluids. 17, 1-14.

Neophytou, A., Mastorakos, E., 2009. Simulations of laminar flame propagation in droplet mists. Combust. Flame 156, 1627-1640.

Neophytou, A., Mastorakos, E., Cant, R.S., 2012. The internal structure of igniting turbulent sprays as revealed by complex chemistry DNS. Combust. Flame 159, 641-664.

Neophytou, A., Mastorakos, E., Cant, R.S., 2010. DNS of spark ignition and edge flame propagation in turbulent droplet-laden mixing layers. Combust. Flame 157, 1071-1086.

Nivarti, G. V., Cant, R. S., 2017. Direct Numerical Simulation of the bending effect in turbulent. premixed flames, Proc. Combust. Inst., 36, 1903-1910.

Ozel Erol, G., Hasslberger, J., Klein, M., Chakraborty, N., 2018. A direct numerical simulation analysis of spherically expanding turbulent flames in fuel droplet-mists for an overall equivalence ratio of unity. Phys. Fluids 086104.

Ozel Erol, G., Hasslberger, J., Klein, M., Chakraborty, N., 2019. A Direct Numerical Simulation investigation of spherically expanding flames propagating in fuel droplet-mists for different droplet diameters and overall equivalence ratios. Combust. Sci. Technol., https://doi.org/10.1080/00102202.2019.1678373.

Ozel Erol, G., Hasslberger, J., Klein, M., Chakraborty, N., 2019. Propagation of spherically expanding turbulent flames into fuel droplet-mists. Flow Turb. Combust.,103, 913-941.

Peters, N., Terhoeven, P., Chen, J.H., and Echekki, T., 1998, Statistics of flame displacement speeds from computations of 2-D unsteady methane-air flames. Proc. Combust. Inst. 27,833839. 
Pillai, A.L., Kurose, R., 2018, Numerical investigation of combustion noise in an open turbulent spray flame. Appl. Acoust., 133, 16-27.

Pillai, A.L., Kurose, R., 2019, Combustion noise analysis of a turbulent spray flame using a hybrid DNS/APE-RF approach. Combust. Flame, 200, 168-91.

Poinsot, T.J., Echekki, T., Mungal, M.G., 1992. A study of the laminar flame tip and implications for premixed turbulent combustion. Combust. Sci. Technol. 81, 45-73.

Polymeropoulos, C.E., 1984. Flame propagation in aerosols of fuel droplets, fuel vapor and air. Combust. Sci. Technol. 40, 217-232.

Reveillon, J., and Vervisch, L., 2005. Analysis of weakly turbulent dilute-spray flames and spray combustion regimes, J. Fluid Mech. 537, 317-347.

Reveillon, J., Demoulin, F.X., 2007. Evaporating droplets in turbulent reacting flows. Proc. Combust. Inst. 31, 2319-2326.

Rotexo-Softpredict-Cosilab, GmbH and Co. KG Bad Zwischenahn, Germany.

Sattler, S. S., Knaus, D. S., Gouldin, F.C., 2002. Determination of three-dimensional flamelet orientation distributions in turbulent v-flames from two-dimensional image data. Proc. Combust. Inst. 29, 1785-1792.

Schroll, P., Wandel, A.P., Cant, R.S., Mastorakos, E., 2009. Direct numerical simulations of autoignition in turbulent two-phase flows. Proc. Combust. Inst. 32, 2275-2282.

Shepherd, I. G., 1996. Flame surface density and burning rate in premixed turbulent flames, Proc. Combust. Inst. 26, 373-379.

Swaminathan, N., Bray, K.N.C., 2011. Turbulent Premixed Flames, Cambridge University Press, NewYork, USA, p. 5.

Silverman, I., Greenberg, J.B., Tambour, Y., 1993. Stoichiometry and polydisperse effects in premixed spray flames. Combust. Flame 93, 97-118.

Smith, K.O., Gouldin, F.C., 1978. Experimental Investigation of Flow Turbulence Effects on 
Premixed Methane-Air Flames. Prog. Astronaut. Aeronaut. 58, 37-54.

Sponfeldner, T., Boxx, I., Beyrau, F., Hardalupas, Y., Meier, W., Taylor, A. M. K. P., 2015. On the alignment of fluid-dynamic principal strain-rates with the 3D flamelet-normal in a premixed turbulent V-flame. Proc. Combust. Inst. 35, 1269-1276.

Sreedhara, S., Huh, K.Y., 2007. Conditional statistics of nonreacting and reacting sprays in turbulent flows by direct numerical simulation. Proc. Combust. Inst. 31, 2335-2342.

Szekely, G.A., Faeth, G.M., 1983. Effects of envelope flames on drop gasification rates in turbulent diffusion flames. Combust. Flame 49, 255-259.

Tarrazo, E.F., Sánchez, A.L., Liñán, A., Williams, F.A., 2006. A simple one-step chemistry model for partially premixed hydrocarbon combustion. Combust. Flame 147, 32-38.

Taylor, G.I. 1922. Diffusion by continuous movements. Proc. Lond. Math. Soc. 20, 196-212. Trouvé, A., and Poinsot, T., 1994. The evolution equation for flame surface density in turbulent premixed combustion, J. Fluid Mech., 278, 1-31.

Vena, P.C., Deschamps, B., Smallwood, G. J., Johnson, M. R., 2011. Equivalence ratio gradient effects on flame front topology in a stratified iso-octane/air turbulent V-flame. Proc. Combust. Inst. 33, 1551-1558.

Vena, P.C., Deschamps, B., Guo, H., Smallwood, G. J., Johnson, M. R., 2015. Heat release rate variations in a globally stoichiometric, stratified iso-octane/air turbulent V-flame. Combust. Flame 162, 944-959.

Veynante, D., Duclos, J. M., Piana, J., 1994. Experimental analysis of flamelet models for premixed turbulent combustion. Proc. Combust. Inst. 25, 1249-1256.

Veynante, D., Piana, J., Duclos, J. M., Martel, C., 1996. Experimental analysis of flame surface density models for premixed turbulent combustion. Proc. Combust. Inst. 26, 413-420.

Wacks, D., Chakraborty, N., Mastorakos, E., 2016. Statistical Analysis of Turbulent FlameDroplet Interaction: A Direct Numerical Simulation Study. Flow, Turbul. Combust. 96, 573- 
607.

Wacks, D., Chakraborty, N., 2016. Flow topology and alignments of scalar gradients and vorticity in turbulent spray flames: A Direct Numerical Simulation analysis. Fuel 184, 922947.

Wandel, A.P., Chakraborty, N., Mastorakos, E., 2009. Direct numerical simulations of turbulent flame expansion in fine sprays. Proc. Combust. Inst. 32, 2283-2290.

Wandel, A.P., 2014. Influence of scalar dissipation on flame success in turbulent sprays with spark ignition. Combust. Flame 161, 2579-2600.

Wang, Y., Rutland, C.J., 2005. Effects of temperature and equivalence ratio on the ignition of n-heptane fuel spray in turbulent flow. Proc. Combust. Inst. 30, 893-900.

Watanabe, H., Kurose, R., Hwang, S.M., Akamatsu, F., 2007. Characteristics of flamelets in spray flames formed in a laminar counterflow. Combust. Flame 148, 234-248.

Wray, A.A., 1990. Minimal storage time advanced schemes for spectral methods. California.

Xia, J., Luo, K.H., 2010. Direct numerical simulation of inert droplet effects on scalar dissipation rate in turbulent reacting and non-reacting shear layers. Flow, Turbul. Combust. 84, $397-422$.

Wu,Y. Y., Chan, C. K., Zhou, L. X., 2011. Large eddy simulation of an ethylene-air turbulent premixed V-flame. J. Comput. Methods Appl. Math 235, 3768-3774.

Yamashita, H., Shimada, M., Takeno, T., 1996. A numerical study on flame stability at the transition point of jet diffusion flames. Proc. Combust. Inst. 26, 27-34.

Zhao, S., Bouali, Z., Mura, A., 2019. Computational investigation of weakly turbulent flame kernel growths in iso-octane droplet clouds in CVC conditions. Flow Turb. Combust. https://doi.org/10.1007 728/s10494-019-00051-x 729. 


\section{FIGURE CAPTIONS}

Figure 1. Variations of the (a) normalised laminar burning velocity $S_{b\left(\phi_{g}\right)} /\left\{S_{b\left(\phi_{g}\right)}\right\}_{\max }$ and (b) normalised adiabatic flame temperature $T_{b\left(\phi_{g}\right)}=\left(T_{a d\left(\phi_{g}\right)}-T_{0}\right) /\left(T_{a d\left(\phi_{g=1}\right)}-T_{0}\right)$ with equivalence ratio $\phi_{g}$ for $n$-heptane obtained from modified single step chemistry (Tarrazo et al., 2006), detailed chemical mechanism (Chaos et al., 2007) and experimental (Kumar et al., 2007) data.

Figure 2. Instantaneous distributions of (a). reaction progress variable, $c$ and (b). gaseous equivalence ratio, $\phi_{g}$ on the central x-y midplane for initial $a_{d} / \delta_{s t}=0.04,0.05,0.06$ at $t=$ $2.0 t_{\text {flow }}$. Blue lines show $c=0.1,0.5$ and 0.9 contours from outer to the inner periphery and grey dots show the droplets residing on the plane (not to the scale).

Figure 3. PDFs of $\phi_{g}$ in the region corresponding to $0.01 \leq c \leq 0.99$ at different locations $A$,

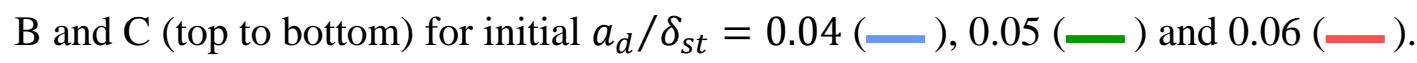

Figure 4. PDFs of $c$ conditional on $\tilde{c}=0.1,0.5,0.9$ at different locations A, B and C (top to bottom) for initial $a_{d} / \delta_{s t}=0.04(\longleftarrow), 0.05(-)$ and $0.06(-)$.

Figure 5. (a) Contours of $\tilde{c}=0.1$ (solid line), 0.5 (dashed line) and 0.9 (dotted line) contours for gaseous premixed ( $\_$) case and droplet cases with initial droplet diameter $a_{d} / \delta_{s t}=0.04$ (—), $0.05\left(\_\right)$and $0.06\left(\_\right)$. The dashed purple lines show the sampling locations (A, B, C from left to right), and the dotted black line shows the flame centre, (b) Distributions of $\tilde{c}$ at the bottom half of the domain for initial $a_{d} / \delta_{s t}=0.04$ ( $1^{\text {st }}$ row $), 0.05$ ( $2^{\text {nd }}$ row $)$ and $0.06\left(3^{\text {rd }}\right.$ row). Black lines show the contour lines of $\tilde{c}=0.1,0.5$, and 0.9 from outer to the inner periphery and red lines are tangents to contour lines at locations A, B and C (left to right).

Figure 6. PDFs of $\phi_{g}$ conditional on $\tilde{c}=0.1,0.5,0.9$ isosurfaces at different locations A, B and C (top to bottom) for initial $a_{d} / \delta_{s t}=0.04(\longleftarrow), 0.05(\longleftarrow)$ and $0.06(\longleftarrow)$.

Figure 7. Instantaneous views of $c=0.5$ isosurface coloured with $\kappa_{m} \times \delta_{s t}$ values for gaseous 
premixed and spray flames with initial $a_{d} / \delta_{s t}=0.04,0.05,0.06$ at $t=2.0 t_{\text {flow }}$.

Figure 8. PDFs of $\kappa_{m} \times \delta_{s t}$ of the $c=0.8$ isosurface at locations A, B and C (top to bottom) for gaseous premixed ( $(-)$ and spray $V$-shaped spray flames with initial $a_{d} / \delta_{s t}=0.04(-)$, $0.05(-)$ and $0.06(-)$.

Figure 9. Percentage of heat release arising from premixed (for initial $a_{d} / \delta_{s t}=0.04(\square), 0.05$ ( $\square), 0.06(\square)$ ) and non-premixed (for initial $a_{d} / \delta_{s t}=0.04(\square), 0.05(\square), 0.06(\square)$ ) modes of combustion at locations $\mathrm{A}, \mathrm{B}$ and $\mathrm{C}$ (left to right).

Figure 10. Variations of mean values of $\dot{w}_{c}$ (solid line), $\nabla \cdot(\rho D \nabla c)$ (line with star marker), $\dot{A}_{c}$ (dotted line), $\dot{S}_{l i q, c}$ (triangle marker), $\rho S_{d}|\nabla c|$ (dashed line) and $\rho_{0} S_{b, s t}|\nabla c|$ (circle) conditional on $c$ for premixed gaseous ( $(-)$ ) and droplet cases with initial $a_{d} / \delta_{s t}=0.04(-), 0.05(-)$ and $0.06(-)$ at locations A,B, C (top to bottom). All terms are normalised by $\delta_{s t} / \rho_{0} S_{b, s t}$.

Figure 11. The mean values of $S_{c} / S_{b, s t}, S_{d}^{*} / S_{b, s t}, S_{c} / S_{b\left(\left\langle\phi_{g}\right\rangle\right)}$ and $S_{d}^{*} / S_{b\left(\left\langle\phi_{g}\right\rangle\right)}$ on the $c=0.8$ isosurface at locations A-C. 